\title{
The Quantum Weak Coupling Limit (II): Langevin Equation and Finite Temperature Case
}

\author{
By \\ Luigi ACCARDI*, Alberto FRIGERIO** \\ and Yun G. LU***
}

\begin{abstract}
We complete the program started in [4] by proving that, in the weak coupling limit, the matrix elements, in the collective coherent vectors, of the Heisenberg evolved of an observable of a system coupled to a quasi-free reservoir through a laser type interaction, converge to the matrix elements of a quantum stochastic process satisfying a quantum Langevin equation driven by a quantum Brownian motion. Our results apply to an arbitrary quasi-free reservoir so, in particular, the finite temperature case is included.
\end{abstract}

\section{§o. Introduction}

In the present paper, the notations and the model will be the same as in [4]. Namely, $H_{0}$ (the system space) and $H_{1}$ (the one-particle space of the reservoir) are Hilbert spaces, $Q \geq 1$ is an operator on $H_{1}, W\left(H_{1}\right)$ is the Weyl-algebra on $H_{1}, \varphi_{Q}$ is the mean zero, gauge invariant quasi-free state on $W\left(H_{1}\right)$, with covariance $Q$, i.e.,

$$
\varphi_{Q}(W(f))=\exp \left(-\frac{1}{2}\langle f, Q f\rangle\right), \quad f \in H_{1}
$$

As in [4] we shall use the following notations: $\left\{\mathscr{H}_{Q}, \pi_{Q}, \Phi_{Q}\right\}$ is the GNS triple of $\left(W\left(H_{1}\right), \varphi_{Q}\right)$; we denote $\pi_{Q}(W(\cdot))$ by $W_{Q}(\cdot)$ and $A_{Q}(f), A_{Q}^{+}(f),\left(f \in H_{1}\right)$ the associated annihilation and creation operators, $H_{R}$ is the free Hamiltonian of the reservoir, $H_{S}$ the free Hamiltonian of the system and

$$
H^{(\lambda)}=H_{S} \otimes 1+1 \otimes H_{R}+\lambda V
$$

with

Communicated by H. Araki, October 7, 1989. Revised November 12,

1991, May 23 and November 10, 1994 and January 30, 1995.

1991 Mathematics Subject Classification(s): 81 C99

Centro Matematico V. Volterra Universit`s digli Studi di Roma Tor Vergata

' Dipartimento di Matematica e Informatica Università di Udine

“Ceintro Matematico V. Voltera Università digli Studi di Roma Tor Vergata

On leeave of absence from Beijing Normal University 


$$
V=-\frac{1}{i}\left(D \otimes A_{Q}^{+}(g)-D^{+} \otimes A_{Q}(g)\right) ; \quad H_{R}=d \Gamma(-H)
$$

is the total Hamiltonian of the composite system. Here $H$ is a self-adjoint operator on $H_{1}$ such that $S_{t}^{0}=e^{-t t H}$ commutes with $Q$ and $D$ is a bounded operator on $H_{0}$ such that (rotating wave approximation)

$$
A d e^{-\imath t H_{S}}(D)=e^{-\imath \omega_{0} t} D, \quad \omega_{0}>0
$$

The evolution operator in interaction representation (wave operator at time $t$ ) is

$$
U^{(\lambda)}(t)=e^{t t H^{(0)}} e^{-t t H^{(\lambda)}}
$$

with

$$
H^{(0)}=H_{S} \otimes 1+1 \otimes H_{R}
$$

and satisfies, weakly on the domain of coherent vectors, the equation

$$
\frac{d}{d t} U^{(\lambda)}(t)=\frac{1}{i} \lambda V(t) U^{(\lambda)}(t)
$$

where,

$$
\begin{aligned}
V(t)= & -\frac{1}{i}\left(D \otimes e^{-i \omega_{0} t} A^{+}\left(S_{t}^{0} g\right)-D^{+} \otimes e^{i \omega_{0} t} A\left(S_{t}^{0} g\right)\right) \\
& =-\frac{1}{i}\left(D \otimes A^{+}\left(S_{t} g\right)-D^{+} \otimes A\left(S_{t} g\right)\right)
\end{aligned}
$$

and

$$
S_{l}=e^{-\imath \omega_{01} t} S_{t}^{0}, \quad t \in \mathbb{R}
$$

In [4], we have shown that, in the Fock representation i.e. for $Q=1$, the limit

$$
\lim _{\lambda \rightarrow 0}\left\langle u \otimes W_{Q}\left(\lambda \int_{S_{1} / \lambda^{2}}^{T_{1} / \lambda^{2}} S_{u} f_{1} d u\right) \Phi_{Q}, U^{(\lambda)}\left(t / \lambda^{2}\right) v \otimes W_{Q}\left(\lambda \int_{S_{2} / \lambda^{2}}^{T_{2} / \lambda^{2}} S_{u} f_{2} d u\right) \Phi_{Q}\right\rangle
$$

exists and is equal to

$$
\left\langle u \otimes W\left(\chi_{\left|S_{1}, T_{1}\right|} \otimes f_{1}\right) \Psi, U(t) \cdot v \otimes W\left(\chi_{\left|S_{2}, T_{2}\right|} \otimes f_{2}\right) \Psi\right\rangle
$$

where, $\left\{\mathbb{X}, \Psi, W\left(\chi_{|S . T|} \otimes f\right)\right\}$ is the Fock Brownian Motion on $L^{2}(\mathbb{R}, d t ; \not y)$. Moreover, from Theorem (II.) in [4], we know that $U(t)$ satisfies the quantum stochastic differential equation,

$$
U(t)=1+\int_{0}^{t}\left(D \otimes d A_{S}^{+}(g)-D^{+} \otimes d A_{S}(g)-(g \mid g)_{-} D^{+} D \otimes 1 d s\right) U(s)
$$

where, $A_{S}(g):=A\left(\chi_{[0,1]} \otimes g\right)$.

The first result of the present paper, is the study of the limit (0.7) for $Q \neq 1$ and the deduction of the corresponding quantum stochastic differential equation. 
(cf. Theorem (1.7) below) The application of this extension, to a system $S$ interacting with a free Boson gas in equilibrium, was discussed without proofs in Section (7.) of [4].

The main new feature in the proof of this result, with respect to [4] is the more complex structure of the negligible and non negligible terms, due to the doubling of the space (with respect to the Fock case).

Most of the work of Section (1.) is devoted to the proof that the estimates of [4] are sufficient to guarantee the convergence to zero of the so-called type II terms and to make esplicit the additional term, in the quantum stochastic equation, due to the fact that the state is non-Fock.

The second new result, both in the Fock and in the $Q \neq 1$ case, is that the limit

$$
\begin{gathered}
\lim _{\lambda \rightarrow 0}\left\langle u \otimes W_{Q}\left(\lambda \int_{S_{1} / \lambda^{2}}^{T_{1} / \lambda^{2}} S_{u} f_{1} d u\right) \Phi_{Q}, U^{(\lambda)}\left(t / \lambda^{2}\right)(X \otimes 1) U^{(\lambda)}\left(t / \lambda^{2}\right)\right. \\
\left.v \otimes W_{Q}\left(\lambda \int_{S_{2} / \lambda^{2}}^{T_{2} / \lambda^{2}} S_{u} f_{2} d u\right) \Phi_{Q}\right\rangle
\end{gathered}
$$

exists for any $X \in B\left(H_{0}\right)$ and is equal to (in the same notations as (0.8))

$$
\left\langle u \otimes W\left(\chi_{\left[S_{1}, T_{1}\right]} \otimes f_{1}\right) \Psi, U(t)(X \otimes 1) U(t)^{\prime} \cdot v \otimes W\left(\chi_{\left[S_{2}, T_{2}\right]} \otimes f_{2}\right) \Psi\right\rangle
$$

where, instead of (0.9), $U(t)$ satisfies the equation (1.45).

This is the main result of the present paper and corresponds to Theorem III, stated without proof in [4]. Its meaning is that the family of bounded quantum stochastic processes

$$
X^{(\lambda)}(t):=U^{(\lambda)}(t)(X \otimes 1) U^{(\lambda)}(t)
$$

satisfying an ordinary Heisenberg equation in interaction representation, converges, weakly in the sense of matrix elements (cf. Definition (2.2) of [4]), to the process

$$
X(t):=U(t)(X \otimes 1) U(t)
$$

satisfying the quantum Langevin equations (4.4a), (4.4b). The main difficulties in the passage from the equations for $U(t)$ (i.e. (0.9) and (1.45)) to the Langevin equations (4.4a) and (4.4b), are all present in the Fock case. Once these have been settled and the result of Theorem (1.7) has been established, the extension of the Langevin equation from the Fock to the finite temperature case can be obtained by some natural (although lengthy) modifications which make use of the ideas developed in Sections (.1) of the present paper. For this reason we do not include the proof of Theorem (4.2).

Throughout this paper, when a proof is a simple adaptation of pervious results, we have barely mentioned the salient points. 
A quantum Langevin equation of different type for a restricted set of observables was deduced, with different techniques, in [6].

It will be clear from the proof that the above mentioned result is by no means an easy corollary of Theorem II of [4], even if the basic estimates of that paper will be constantly used here. On the other hand such a result is needed if we want to include in the present theory all the previous results on the master equation [6], [9], [10]. Given the above result, this inclusion is a simple corollary of the quantum Feynman-Kac formula [1] (for the connection between the quantum Feynman-Kac formula and the Langevin equation, see [2]).

As in [4], we suppose that there exists a non-zero subspace $K \subseteq \operatorname{Dom}(Q$ ) (in all the examples it will be a dense subspace) such that

$$
\int_{R}\left\langle\left\langle f_{1}, S_{t} f_{2}\right\rangle\right| d t<\infty, \quad \forall f_{1}, f_{2} \in K
$$

This condition implies that the sesquilinear form

$$
f_{1}, f_{2} \in K \rightarrow\left(f_{1} \mid f_{2}\right):=\int_{R}\left|\left\langle f_{1}, S_{t} f_{2}\right\rangle\right| d t
$$

defines a pre-scalar product on $K$. We shall still denote by $K$ (or $(K,(+))$ if confusion can arise) the associated Hilbert space, i.e. the completion of the quotient of $K$ by the zero norm elements in the scalar product $(0.12)$. The scalar product on $K$ will still be denoted by $(\cdot)$. In the following we shall use, without proof, the following three results from [4].

Lemma (0.1). For each $g \in \operatorname{Dom}(Q)$ and for any $-\infty<S \leq T<+\infty$, the integral

$$
\int_{S}^{T} S_{t} g d t
$$

is well defined and belongs to $\operatorname{Dom}(Q)$, moreover,

$$
Q \int_{S}^{T} S_{t} g d t=\int_{S}^{T} Q S_{t} g d t=\int_{S}^{T} S_{t} Q g d t
$$

Lemma (0.2). For each pair $f, g \in K$ satisfying (0.10) and for any $S_{1}, T_{1}$, $S_{2}, T_{2} \in \mathbb{R}\left(S, \leq T_{1}, j=1,2\right)$, one has

$$
\lim _{\lambda \rightarrow 0}\left\langle\lambda \int_{S_{1} / \lambda^{2}}^{T_{1} / \lambda^{2}} S_{u} f d u, \lambda \int_{S_{2} / \lambda^{2}}^{T_{2} / \lambda^{2}} S_{u} g d u\right\rangle=\left\langle\chi_{\left|S_{1}, T_{1}\right|}, \chi_{\left|S_{2}, T_{2}\right|}\right\rangle \int_{\mathbf{R}}\left\langle f_{1}, S_{1} g\right\rangle d t
$$

where, the scalar product of the characteristic functions are meant in $L^{2}(\mathbb{R})$ and the limit is uniform for $S_{1}, T_{1}, S_{2}, T_{2}$ in a bounded set of $\mathbb{R}$. 
Lemma (0.3). For each $n \in \mathbf{N}, f_{1}, \cdots, f_{n} \in K, S_{1}, T_{1}, \cdots, S_{n}, T_{n} \in \mathbf{R}, x_{1}, \cdots, x_{n} \in$ $\mathbf{R}$, the limit

$$
\lim _{\lambda \rightarrow 0}\left\langle\Phi_{Q}, W_{Q}\left(x_{1} \lambda \int_{S_{1} / \lambda^{2}}^{T_{1} / \lambda^{2}} S_{u} f_{1} d u\right) \cdots W_{Q}\left(x_{n} \lambda \int_{S_{n} / \lambda^{2}}^{T_{n} / \lambda^{2}} S_{u} f_{n} d u\right) \Phi_{Q}\right\rangle
$$

exists uniformly for $x_{1}, \cdots, x_{n}, S_{1}, \cdots, S_{n}, T_{1}, \cdots, T_{n}$ in a bounded set of $\mathbf{R}$ and it is equal to

$$
\left\langle\Psi_{Q}, W_{Q}\left(x_{1} \chi_{\left[S_{1}, T_{1}\right]} \otimes f_{1}\right) \cdots W_{Q}\left(x_{n} \chi_{\left[S_{n}, T_{n}\right]} \otimes f_{n}\right) \Psi_{Q}\right\rangle
$$

where, $\left\{\mathscr{H}^{\prime}, \Psi_{Q}, W_{Q}\left(\chi_{[S, T]} \otimes f\right)\right\}$ is the $Q$-Brownian Motion on $L^{2}\left(\mathbf{R}, d t ; \mathscr{H}^{\prime}\right)$.

Throughout the paper, we shall use the notations

$$
D_{0}=-D^{+} ; D_{1}=D
$$

and

$$
A^{0}=A ; A^{1}=A^{+}
$$

\section{Acknowledgements}

L. Accardi acknowledges support from Grant AFOSR 870249 and ONR N00014-86-K-0538 through the Center for Mathematical System Theory, University of Florida.

\section{Added in the second revision}

The present paper has undergone two revisions because the authors found some difficulties in explaining that the first part of the paper is devoted to establish the convergence of (0.7) to $(0.8)$ in the quasi-free non-Fock case and the second one to establish the convergence of $(0.10)$ to $(0.10 \mathrm{a})$ in the Fock case. This implied a number of revisions of the introduction which were also delayed by subsequent developments. On the other hand, the statement of the theorems and their proofs have not undergone any revision and they are the same as in the original version of the paper in July 1989.

\section{Added in the third revision}

On request of the referee, we have inserted the proof of the unitarity of the operator $U(t)$ in Theorem (1.7). No other changes were required.

\section{Added in the fourth revision}

On further request of the referee, we correct a statement made in the subsection Added in the second revision. Effectively our claim that the statement of the theorems and their proofs have not undergone any revision and they are the same as in the original version of the paper in July 1989. is not true: we added in Theorem (1.7) the statement. Moreover $U(t)$ is unitary for each $t \geq 0$ and the 
referee asked for its proof. The proof is an application of the standard unitarity condition and of the standard representation of a boson, quasi-free state. In the present (fourth) revision of our paper, no other changes were required by the referee, nor introduced by us.

We take the occasion of this further requirement of revision of the present paper to make esplicit that the subsequent developments mentioned in the subsection Added in the second revision have nothing to do with the present paper. They concern: the complete solution of the low density problem and the related connection with quantum scattering, the discovery that the Wigner-Voiculescu diagrams of the semicircle law arise canonically from the solution of the problem considered here in quantum electrodynamics, without dipole approximation, the applications to quantum chromodynamics, the emergence of quantum noises living on Hilbert modules rather than Hilbert spaces, and the development of the associated stochastic calculus, the applications to the $1 / N$-expansion, the discovery of a new type of semiclassical approximation in quantum field theory, arising from the present limit, and its application to high energy physics, the applications to stochastic bosonization in arbitrary dimensions, ... .

\section{$\S 1$. The Gauge Invariant Quasi Free Case}

For any Hilbert space $H$, denote $H^{\prime}$ the conjugate space of $H$, i.e.

$$
H^{l}=\left(H,\langle\cdot, \cdot\rangle_{l}\right)
$$

where $H^{\imath}$ coincides with $H$ as a set, $\imath: f \in H \rightarrow H$ is the identity map and

$$
\begin{gathered}
l(\lambda f):=\bar{\lambda}_{\imath}(f), f \in H \\
\langle\imath(f), \imath(g)\rangle_{l}:=\langle g, f\rangle, f, g \in H
\end{gathered}
$$

For a linear operator $A: H \rightarrow H$, we write

$$
A^{l}: H^{\imath} \rightarrow H^{l}, A^{l}(l(f)):=\imath(A f), f \in H
$$

Denoting by $\varphi_{F}$ and $\varphi_{F}^{\prime}$ the Fock states on the Weyl algebras $W(H)$ and $W\left(H^{l}\right)$ respectively and $\left\{\mathscr{H}_{F}, \pi_{F}, \Phi_{F}\right\},\left\{\mathscr{H}_{F}^{\prime \prime}, \pi_{F}^{\prime}, \Phi_{F}^{\prime}\right\}$ the corresponding GNS triples, and putting

$$
Q_{+}=\sqrt{\frac{Q+1}{2}}, Q_{-}=\imath \sqrt{\frac{Q-1}{2}}
$$

It follows that, if $\left\{\dddot{M}_{Q}, \pi_{Q}, \Phi_{Q}\right\}$ is the GNS triple of $\left\{W(H), \varphi_{Q}\right\}$, then the map

$$
\begin{aligned}
\pi_{Q}(W(f)) \rightarrow & \pi_{F}\left(W\left(Q_{+} f\right)\right) \otimes \pi_{F}^{\prime}\left(W\left(Q_{-} f\right)\right) \\
& \Phi_{Q} \rightarrow \Phi_{F} \otimes \Phi_{F}^{l}
\end{aligned}
$$


extends to a unitary isomorphism of the triple $\left\{\dddot{K}_{Q}, \pi_{Q}, \Phi_{Q}\right\}$ with the triple $\left\{\mathscr{H}_{F}^{\prime} \otimes \mathscr{H}_{F}^{\prime}, \pi_{F} \otimes \pi_{F}^{i}, \Phi_{F} \otimes \Phi_{F}^{l}\right\}$. In the following, this unitary isomorphism will be denoted by $R$. With this notation, (1.6) becomes:

$$
R \pi_{Q}(W(f)) R^{\prime}=\pi_{F}\left(W\left(Q_{+} f\right)\right) \otimes \pi_{F}^{l}\left(W\left(Q_{-} f\right)\right)
$$

Thus, for the field operators one obtains:

$$
\begin{gathered}
R \pi_{Q}(B(f)) R^{\prime}=\left.\frac{1}{i} \frac{d}{d t} R \pi_{Q}(W(t f)) R^{\prime}\right|_{t=0}=\left.\frac{1}{i} \frac{d}{d t} \pi_{F}\left(W\left(t Q_{+} f\right)\right) \otimes \pi_{F}^{l}\left(W\left(t Q_{-} f\right)\right)\right|_{t=0} \\
=\pi_{F}\left(B\left(Q_{+} f\right)\right) \otimes 1-1 \otimes \pi_{F}^{l}\left(B\left(Q_{-} f\right)\right)
\end{gathered}
$$

Moreover,

$$
R \pi_{Q}(A(f)) R^{\prime}=\pi_{F}\left(A\left(Q_{+} f\right)\right) \otimes 1+1 \otimes \pi_{F}^{l}\left(A^{+}\left(Q_{-} f\right)\right)
$$

From now on, we shall usually omit the map $\pi_{Q}, \pi_{Q}^{l}$ and write simply $W_{Q}, A_{Q}$, $A_{Q}^{+}$for $\pi_{Q}(W), \pi_{Q}(A), \pi_{Q}\left(A^{+}\right)$, or $\pi_{Q}^{l}(W), \pi_{Q}^{\prime}(A), \pi_{Q}^{l}\left(A^{+}\right)$and, if $Q=1$ (Fock case), we simply write $W, A, A^{+}$and we identify $R$ with $1 \otimes R$. With these notations, for any $n \in \mathbb{N}$ and $g_{1}, \cdots, g_{n} \in H_{1}$ one has,

$$
\begin{gathered}
R\left(D \otimes A_{Q}^{+}\left(g_{1}\right)-D^{+} \otimes A_{Q}\left(g_{1}\right) \cdot \cdots \cdot\left(D \otimes A_{Q}^{+}\left(g_{n}\right)-D^{+} \otimes A_{Q}\left(g_{n}\right)\right) R\right. \\
=: R \prod_{k=1}^{n}\left(D \otimes A_{Q}^{+}\left(g_{k}\right)-D^{+} \otimes A_{Q}\left(g_{k}\right)\right) R \\
=\sum_{\varepsilon \in\{0,1\}^{n}} D_{\varepsilon(1)} \cdots D_{\varepsilon(n)} \otimes R A_{Q}^{\varepsilon(1)}\left(g_{1}\right) \cdots A_{Q}^{\varepsilon(n)}\left(g_{n}\right) R \\
=\sum_{\varepsilon \in\{0,1\}^{n}} D_{\varepsilon(1)} \cdots D_{\varepsilon(n)} \otimes\left(A^{\varepsilon(1)}\left(Q_{+} g_{1}\right) \otimes 1+1 \otimes A^{1-\varepsilon(1)}\left(Q_{-} g_{1}\right)\right) \\
\left.\cdots A^{\varepsilon(n)}\left(Q_{+} g_{n}\right) \otimes 1+1 \otimes A^{1-\varepsilon(n)}\left(Q_{-} g_{n}\right)\right) \\
\left.\sum_{\varepsilon \in\{0,1\}^{n}} D_{\varepsilon(1)} \cdots D_{\varepsilon(n)} \otimes A^{\varepsilon(1)}\left(Q_{+} g_{1}\right) \otimes 1\right)^{\sigma(1)} \cdots\left(A^{\varepsilon(1)}\left(Q_{+} g_{n}\right) \otimes 1\right)^{\sigma(n)} \\
\left(1 \otimes A^{1-\varepsilon(1)}\left(Q_{-} g_{1}\right)\right)^{1-\sigma(1)} \cdots\left(1 \otimes A^{1-\varepsilon(n)}\left(Q_{-} g_{n}\right)\right)^{1-\sigma(n)}
\end{gathered}
$$

where, in (1.10), the exponents $\varepsilon(j)$ and $\sigma(j)$ have different meaning, namely:

$$
\begin{aligned}
& A^{\varepsilon}=A \text { if } \varepsilon=0 ;=A^{+} \text {if } \varepsilon=1 \\
& X^{\sigma}=1 \text { if } \sigma=0 ;=X \text { if } \sigma=1
\end{aligned}
$$

The explicit form of the product of creators and annihilators in (1.10) is

$$
\begin{gathered}
\left(A^{\varepsilon(/ 1)}\left(Q_{+} g_{/ 1}\right) \cdot \ldots \cdot A^{\varepsilon(/ h)}\left(Q_{+} g_{/ h}\right) \otimes 1\right) \cdot\left(1 \otimes A^{1-\varepsilon(/ h)}\left(Q_{-} g_{/ i}\right) \cdot\right. \\
\left.\ldots A^{1-\varepsilon\left(l_{n-h}^{i}\right)}\left(Q_{-} g_{l_{n-h}^{-}}\right)\right)
\end{gathered}
$$

Here, for each $k \leq n \in \mathbb{N}, 1 \leq j_{1}<\cdots<j_{k} \leq n$, we have introduced the notation:

$$
\left\{j_{1}^{\iota}, \cdots, j_{n-h}^{\iota}\right\}=\{1, \cdots, n\} \backslash\left\{j_{h}\right\}_{h=1}^{h}
$$


with

$$
j_{1}^{c}<\cdots<j_{n-h}^{c}
$$

Now, notice that each of the two factors in the product (1.13) is of the type (4.8), considered in Lemma (4.1) of [4] and therefore, in the notations (4.8) of [4], and remembering that the multi-index $\varepsilon$ is uniquely determined by the positions of the creation operator, we see that (1.13) takes the form:

$$
\left(I_{g}^{\varepsilon(\jmath)} \otimes 1+I I_{g}^{\varepsilon(\jmath)} \otimes 1\right) \cdot\left(1 \otimes I_{g}^{\varepsilon\left(\jmath^{(}\right)}+1 \otimes I I_{g}^{\varepsilon\left(\jmath^{c}\right)}\right)
$$

with $\varepsilon(j)=\left(\varepsilon\left(j_{1}\right), \cdots, \varepsilon\left(j_{k}\right)\right)$ and $\varepsilon\left(j^{c}\right)=\left(\varepsilon\left(j_{1}^{c}\right), \cdots, \varepsilon\left(j_{n-k}^{c}\right)\right)$. Expanding (1.16) we obtain an expression of the form

$$
I_{g}^{\varepsilon(\jmath)} \otimes I_{g}^{\varepsilon\left(J^{\prime}\right)}+\left(I_{g}^{\varepsilon(\jmath)} \otimes I I_{g}^{\varepsilon\left(J^{c}\right)}+I I_{g}^{\varepsilon(\jmath)} \otimes I_{g}^{\varepsilon\left(J^{c}\right)}+I I_{g}^{\varepsilon(\jmath)} \otimes I I_{g}^{\varepsilon\left(\jmath^{\prime}\right)}\right)
$$

In the following we shall show that only the terms of the form $I_{g}^{\varepsilon(J)} \otimes I_{g}^{\varepsilon\left(I^{\prime}\right)}$ survive in the limit $\lambda \rightarrow 0$ and that the other terms play, in this case, the role played by the terms which, in formula (4.8) of [4], we have called of type $I I$.

In order to achieve this program, let us begin to define the decomposition (1.17) in a precise way. To this goal, taking $g \in H_{1}$, and denoting $g_{k}=S_{t_{k}} g$, one has

$$
R V_{g}\left(t_{1}\right) \cdots V_{q}\left(t_{n}\right) R=R i^{n} \prod_{h=1}^{n}\left(D \otimes A^{+}\left(g_{h}\right)-D^{+} \otimes A\left(g_{h}\right)\right) R
$$

Defining

$$
\begin{gathered}
\Delta_{n}^{(\lambda)}(t):=\lambda^{n} \int_{0}^{t / \lambda^{2}} d t_{1} \int_{0}^{t_{1}} d t_{2} \cdots \int_{0}^{t_{n-1}} d t_{n} \\
\left\langle u \otimes W_{Q}\left(\lambda \int_{S_{1} / \lambda^{2}}^{T_{1} / \lambda^{2}} S_{u} f_{1} d u\right) \Phi_{Q},(-i)^{n} V_{g}\left(t_{1}\right) \cdots V_{g}\left(t_{n}\right) \nu \otimes W_{Q}\left(\lambda \int_{S_{2} / \lambda^{2}}^{T_{2} / \lambda^{2}} S_{u} f_{2} d u\right) \Phi_{Q}\right\rangle
\end{gathered}
$$

and expanding $V_{q}\left(t_{1}\right) \cdots V_{g}\left(t_{n}\right)$ according to $(1.10),(1.13)$ and (1.18) we see that the right hand side of (1.19) is equal to

$$
\begin{aligned}
& \lambda^{n} \int_{0}^{t / \lambda^{2}} d t_{1} \int_{0}^{t_{1}} d t_{2} \cdots \int_{0}^{t_{n-1}} d t_{n}\left\langle u, D_{\varepsilon(1)} \cdots D_{\varepsilon(n)} v\right\rangle \\
& \left\langle W\left(\lambda \int_{S_{1} / \lambda^{2}}^{T_{1} / \lambda^{2}} S_{u} Q_{+} f_{1} d u\right) \Phi_{F} \otimes W\left(\lambda \int_{S_{1} / \lambda^{2}}^{T_{1} / \lambda^{2}} S_{u} Q_{-} f_{1} d u\right) \Phi_{F}^{\prime},\right. \\
& \sum_{\varepsilon \in\{0.1\}^{\prime \prime}} \sum_{k=0}^{n} \sum_{1 \leq \mu_{1}<<l_{k} \leq n} A^{\varepsilon\left(\mu_{1}\right)}\left(Q_{+} g_{h_{1}}\right) \cdots A^{\varepsilon\left(/_{k}\right)}\left(Q_{+} g_{/ k}\right) \otimes \\
& \otimes A^{1-\varepsilon\left(/_{i}^{\prime}\right)}\left(Q_{-} g_{l_{i}^{i}}\right) \cdots A^{1-\varepsilon\left(/_{n-h}^{i}\right)}\left(Q_{-} g_{l_{n-h}^{i}}\right) \\
& \left.W\left(\lambda \int_{S_{2} / \lambda^{2}}^{T_{2} / \lambda^{2}} S_{u} Q_{+} f_{2} d u\right) \Phi_{F} \otimes W\left(\lambda \int_{S_{2} / \lambda^{2}}^{T_{2} / \lambda^{2}} S_{u} Q_{-} f_{2} d u\right) \Phi_{F}^{l}\right\rangle
\end{aligned}
$$




$$
\begin{gathered}
=\sum_{\varepsilon \in[0.1\}^{n}} \sum_{h=0}^{n} \sum_{1 \leq J_{1}<<J_{k} \leq n}\left\langle u, D_{\varepsilon(1)} \cdots D_{\varepsilon(n)} v\right\rangle \\
\lambda^{n} \int_{0}^{t / \lambda^{2}} d t_{1} \int_{0}^{t_{1}} d t_{2} \cdots \int_{0}^{t_{n-1}} d t_{n}\left\langle W\left(\lambda \int_{S_{1} / \lambda^{2}}^{T_{1} / \lambda^{2}} S_{u} Q_{+} f_{1} d u\right) \Phi_{F},\right. \\
\left.A^{\varepsilon\left(\mu_{1}\right)}\left(Q_{+} g_{J_{1}}\right) \cdots A^{\varepsilon\left(J_{k}\right)}\left(Q_{+} g_{J_{k}}\right) W\left(\lambda \int_{S_{2} / \lambda^{2}}^{T_{2} / \lambda^{2}} S_{u} Q_{+} f_{2} d u\right) \Phi_{F}\right\rangle \\
\left\langle W\left(\lambda \int_{S_{1} / \lambda^{2}}^{T_{1} / \lambda^{2}} S_{u} Q_{-} f_{1} d u\right) \Phi_{F}^{l}, A^{1-\varepsilon\left(h_{1}^{i}\right)}\left(Q_{-} g_{l_{1}^{\prime}}\right) \cdots A^{1-\varepsilon\left(J_{n-h}^{l}\right)}\left(Q_{-} g_{J_{n-h}^{\prime}}\right)\right. \\
\left.W\left(\lambda \int_{S_{2} / \lambda^{2}}^{T_{2} / \lambda^{2}} S_{u l} Q_{-} f_{2} d u\right) \Phi_{F}^{l}\right\rangle_{l}
\end{gathered}
$$

Now, if for each of the two scalar products in (1.20) we separate the terms of type I and those of type II, we obtain four types of terms. If we split them according to the decomposition (1.17), we see that the expression (1.20) above splits into a sum of two types of terms

$$
\Delta_{n}^{(\lambda)}(1)+\Delta_{n}^{(\lambda)}(2)
$$

where, $\Delta_{n}^{(\lambda)}(1)$ corresponds, in the notations of $(1.17)$ to the products $I_{g}^{\varepsilon(\jmath)} \otimes I_{g}^{\varepsilon\left(l^{\prime}\right)}$ and has the form

$$
\begin{aligned}
& \Delta_{n}^{(\lambda)}(1):=\sum_{k=0}^{n} \sum_{1 \leq \mu_{1}<<l_{k} \leq n} \lambda^{n} \int_{0}^{t / \lambda=} d t_{1} \int_{0}^{t_{1}} d t_{2} \cdots \int_{0}^{t_{n-1}} d t_{n}
\end{aligned}
$$

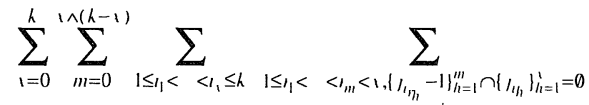

$$
\begin{aligned}
& \left\{h_{h_{h}}-1\right\}_{h=1}^{m} \subset\left\{h_{h h}\right\}_{h=1}^{k} \backslash\left\{h_{h_{h}},\right\}_{h=1}^{m} \\
& \left\langle W\left(\lambda \int_{S_{1} / \lambda^{2}}^{T_{1} / \lambda^{2}} S_{u} Q_{+} f_{1} d u\right) \Phi_{F}, \prod_{h=1}^{m}\left\langle S_{t_{h_{h}}-1} Q_{+} g, S_{t_{h_{h}}} Q_{+} g\right\rangle\right.
\end{aligned}
$$

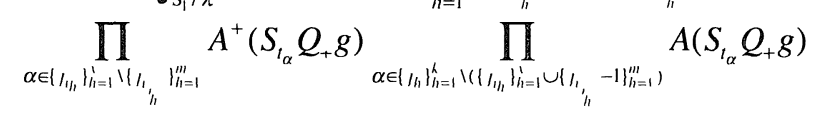

$$
\begin{aligned}
& \left.W\left(\lambda \int_{S_{2} / \lambda^{2}}^{T_{2} / \lambda^{2}} S_{u} Q_{+} f_{2} d u\right) \Phi_{F}\right\rangle
\end{aligned}
$$

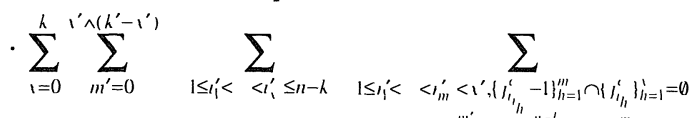

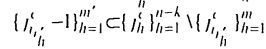

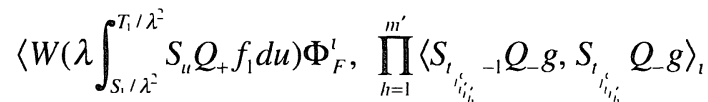

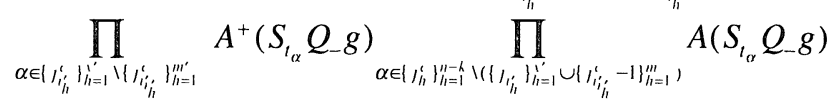

$$
\begin{aligned}
& \left.W\left(\lambda \int_{S_{2} / \lambda^{2}}^{T_{2} / \lambda^{2}} S_{\| 1} Q_{-} f_{2} d u\right) \Phi_{F}^{l}\right\rangle_{1} \cdot\left\langle u, D_{n}\left(j_{1}, \cdots, j_{h} ; i_{1}, \cdots, i_{1} ; i_{1}^{\prime}, \cdots, i_{1}^{\prime}\right) v\right\rangle
\end{aligned}
$$


where we introduced the notation

$$
D_{n}\left(j_{1}, \cdots, j_{k} ; i_{1}, \cdots, i_{1} ; i_{1}^{\prime}, \cdots, i_{1}^{\prime}\right)=D_{\varepsilon(1)} \cdots D_{\varepsilon(n)}
$$

with

$$
\varepsilon(j)=\left\{\begin{array}{l}
1, \text { if } j \in\left\{j_{l_{h}}\right\}_{h=1}^{\prime} \cup\left(\left\{j_{h}^{c}\right\}_{h=1}^{n-h} \backslash\left\{j_{l_{h}^{\prime}}^{c}\right\}_{h=1}^{\prime \prime}\right) ; \\
0, \text { otherwise. }
\end{array}\right.
$$

The term $\Delta_{n}^{(\lambda)}(2)$ in the notations of $(1.21)$ is equal to

$$
\begin{gathered}
\Delta_{n}^{(\lambda)}(2)=\sum_{h=0}^{n} \sum_{1 \leq J_{1}<<l_{l} \leq n} \lambda^{n} \int_{0}^{t / \lambda^{2}} d t_{1} \int_{0}^{t_{1}} d t_{2} \cdots \int_{0}^{t_{n-1}} d t_{n} \\
\left\langle W\left(\lambda \int_{S_{1} / \lambda^{2}}^{T_{1} / \lambda^{2}} S_{u} Q_{+} f_{1} d u\right) \Phi_{F} \otimes W\left(\lambda \int_{S_{1} / \lambda^{2}}^{T_{1} / \lambda^{2}} S_{u} Q_{-} f_{1} d u\right) \Phi_{F}^{l},\right. \\
\left(I_{q}^{\varepsilon(\prime)} \otimes I I_{q}^{\varepsilon(\prime)}+I I_{q}^{\varepsilon(\prime)} \otimes I_{g}^{\varepsilon\left(\prime^{\prime}\right)}+I I_{g}^{\varepsilon(\jmath)} \otimes I I_{g}^{\varepsilon\left(\prime^{\prime}\right)}\right) \\
\left.W\left(\lambda \int_{S_{2} / \lambda^{2}}^{T_{2} / \lambda^{2}} S_{u} Q_{+} f_{2} d u\right) \Phi_{F} \otimes W\left(\lambda \int_{S_{2} / \lambda^{2}}^{T_{2} / \lambda^{2}} S_{u} Q_{-} f_{2} d u\right) \Phi_{F}^{l}\right\rangle \\
=:[I \otimes I I]+[I I \otimes I]+[I I \otimes I I]
\end{gathered}
$$

with the following form:

$$
\begin{aligned}
& {[I \otimes I I]=\sum_{k=0}^{n} \sum_{1 \leq J_{1}<<\mu_{k} \leq n} \lambda^{n} \int_{0}^{t^{\prime} \lambda^{2}} d t_{1} \int_{0}^{t_{1}} d t_{2} \cdots \int_{0}^{t_{n-1}} d t_{n}}
\end{aligned}
$$

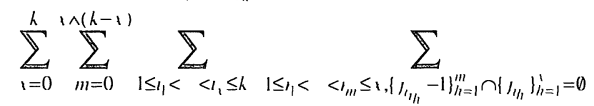

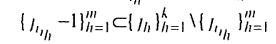

$$
\begin{aligned}
& \left\langle W\left(\lambda \int_{S_{1} / \lambda^{2}}^{T_{1} / \lambda^{2}} S_{u} Q_{+} f_{1} d u\right) \Phi_{F}, \quad \prod_{h=1}^{m}\left\langle S_{t_{t^{\prime \prime},-1}} Q_{+} g, S_{t_{l^{\prime \prime}, h}} Q_{+} g\right\rangle\right.
\end{aligned}
$$

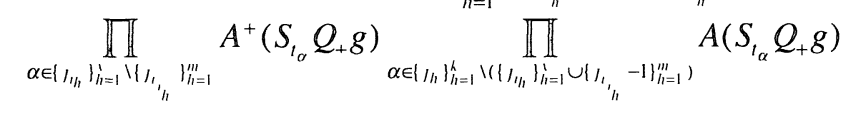

$$
\begin{aligned}
& \left.W\left(\lambda \int_{S_{2} / \lambda^{2}}^{T_{2} / \lambda^{2}} S_{u} Q_{+} f_{2} d u\right) \Phi_{F}\right\rangle \\
& \sum_{i^{\prime}=0}^{n-h} \sum_{m^{\prime}=0}^{i^{\prime} n\left(n-h-1^{\prime}\right)} \sum_{1 \leq i_{1}^{\prime}<<<i_{1}^{\prime} \leq n-h} \sum_{\left(p_{1}^{\prime}, q_{1}^{\prime}, p_{m}^{\prime}, q_{m}^{\prime}\right)}^{\prime}
\end{aligned}
$$

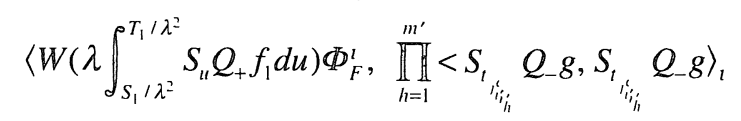

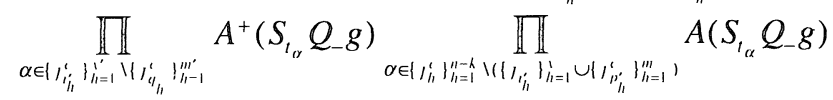

$$
\begin{aligned}
& \left.W\left(\lambda \int_{S_{2} / \lambda^{2}}^{T_{2} / \lambda^{2}} \quad S_{u} Q_{-} f_{2} d u\right) \Phi_{F}^{l}\right\rangle_{l}\left\langle u, D_{n}\left(j_{1}, \cdots, j_{k} ; i_{1}, \cdots, i_{1} ; i_{1}^{\prime}, \cdots, i_{1}^{\prime}\right) v\right\rangle \\
& {[I I \otimes I]=\sum_{h=0}^{n} \sum_{1 \leq \mu_{1}<<<l_{h} \leq n} \lambda^{n} \int_{0}^{t^{\prime} / \lambda^{2}} d t_{1} \int_{0}^{t_{1}} d t_{2} \cdots \int_{0}^{t_{n-1}} d t_{n}}
\end{aligned}
$$




$$
\begin{aligned}
& \sum_{i=0}^{h} \sum_{m=0}^{1 \wedge(h-1)} \sum_{1 \leq 1_{1}<<l_{1} \leq h} \sum_{\left(p_{1}, q_{1}, p_{m}, q_{m}\right)}^{\prime} \\
& \left\langle W\left(\lambda \int_{S_{1} / \lambda^{2}}^{T_{1} / \lambda^{2}} S_{u} Q_{+} f_{1} d u\right) \Phi_{F}, \prod_{h=1}^{m}\left\langle S_{t_{J_{p}}} Q_{+} g, S_{t_{t u_{h}}} Q_{+} g\right\rangle\right.
\end{aligned}
$$

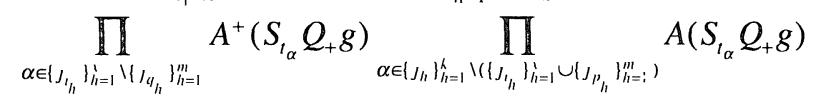

$$
\begin{aligned}
& \left.W\left(\lambda \int_{S_{2} / \lambda^{2}}^{T_{2} / \lambda^{2}} S_{u} Q_{+} f_{2} d u\right) \Phi_{F}\right\rangle
\end{aligned}
$$

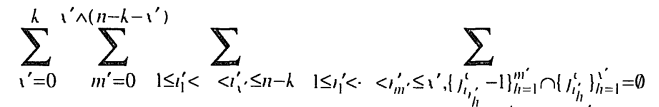

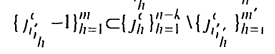

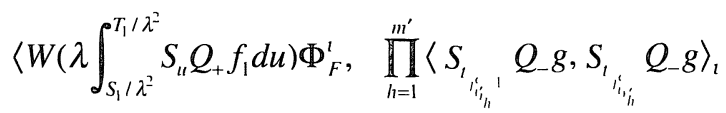

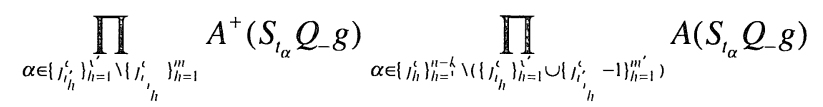

$$
\begin{aligned}
& \left.W\left(\lambda \int_{S_{2} / \lambda^{2}}^{T_{2} / \lambda^{2}} S_{u} Q_{-} f_{2} d u\right) \Phi_{F}^{\prime}\right\rangle_{l}\left\langle u, D_{n}\left(j_{1}, \cdots, j_{k} ; i_{1}, \cdots, i_{1} ; i_{1}^{\prime}, \cdots, i_{1^{\prime}}^{\prime}\right) v\right\rangle \\
& {[I \otimes I I]=\sum_{h=0}^{n} \sum_{I_{1}<<<l_{k} \leq n} \lambda^{n} \int_{0}^{t / \lambda^{2}} d t_{1} \int_{0}^{t_{1}} d t_{2} \cdots \int_{0}^{t_{n-1}} d t_{n}} \\
& \sum_{i=0}^{h} \sum_{m=0}^{i n k-1)} \sum_{1 \leq 1_{1}<<l_{1} \leq k} \sum_{\left(p_{1}, q_{1}, p_{m}, q_{m}\right)}^{\prime} \\
& \left\langle W\left(\lambda \int_{S_{1} / \lambda^{2}}^{T_{1} / \lambda^{2}} S_{u} Q_{+} f_{1} d u\right) \Phi_{F}, \prod_{h=1}^{m}\left\langle S_{t_{t p_{h}}} Q_{+} g, S_{t_{t q_{h}}} Q_{+} g\right\rangle\right.
\end{aligned}
$$

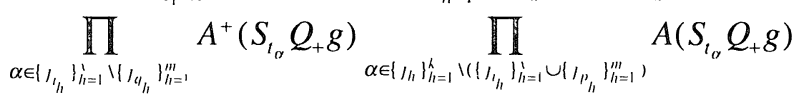

$$
\begin{aligned}
& \left.W\left(\lambda \int_{S_{2} / \lambda^{2}}^{T_{2} / \lambda^{2}} S_{u} Q_{+} f_{2} d u\right) \Phi_{F}\right\rangle \\
& \sum_{1^{\prime}=0}^{n-h} \sum_{m^{\prime}=0}^{i^{\prime} \wedge\left(n-h-1^{\prime}\right)} \sum_{1 \leq i_{1}^{\prime}<<i_{1}^{\prime} \leq n-h} \sum_{\left(p_{1}^{\prime}, q_{1}^{\prime}, p_{m,}^{\prime}, q_{m}^{\prime}\right)}^{\prime} \\
& \left\langle W\left(\lambda \int_{S_{1} / \lambda^{2}}^{T_{1} / \lambda^{2}} S_{u} Q_{-} f_{1} d u\right) \Phi_{F}^{l}, \quad \prod_{h=1}^{m^{\prime}}\left\langle S_{i_{i p_{h}}} Q_{-} g, S_{i_{i t_{h}}} Q_{-} g\right\rangle_{1}\right.
\end{aligned}
$$

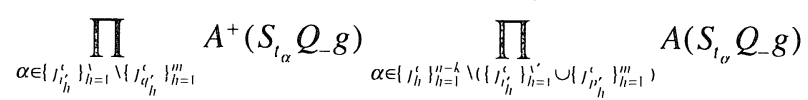

$$
\begin{aligned}
& \left.W\left(\lambda \int_{S_{2} / \lambda^{2}}^{T_{2} / \lambda^{2}} S_{u} Q_{-} f_{2} d u\right) \Phi_{F}^{l}\right\rangle_{l}\left\langle u, D_{n}\left(j_{1}, \cdots, j_{k} ; i_{1}, \cdots, i_{1} ; i_{1}^{\prime}, \cdots, i_{1}^{\prime}\right) v\right\rangle
\end{aligned}
$$

where, $\sum_{\left(p_{1}, q_{1}, \ldots p_{m}, q_{m}\right)}^{\prime}$ is the sum for all $p_{1}, \cdots, p_{m}$ such that

(i) $\left|\left\{p_{h}\right\}_{h=1}^{\prime m}\right|=m$ 
(ii) $p_{h}<q_{h}$ for each $h=1, \cdots, m$ and $j_{p_{h}}<j_{q_{h}}-1$ for some $h=1, \cdots, m$

The sum $\sum_{\left(p_{1}^{\prime}, q_{1}^{\prime}, p_{m^{\prime}}^{\prime}, q_{m^{\prime}}^{\prime}\right)}^{\prime}$ has the analogous meaning.

The length and the complexity of the formulas (1.22), (1.25) should not obscure the basic conceptual difference between these two kinds of terms: in the terms of type I (formula (1.22)) all the scalar products $\left\langle S_{t_{1}} F, S_{t_{\mathrm{h}}} G\right\rangle$ correspond to time indices such that $t_{k}=t_{j+1}$. In the terms of type II (formula (1.25)) there are some scalar products of the form $\left\langle S_{t_{1}} F, S_{t_{k}} G\right\rangle$ with $|j-k|>1$. The basic intuition of the weak coupling phenomenon is that this second kind of terms go to zero. The main estimate that allows to turn this intuition into a precise statement is the same as in the Fock case, only that here the combinatorics is more complex due to the doubling of the Fock space.

As an help for the reader's intuition we summarize the meaning of the (unfortunately many) indices which appear in the formulas (1.22), (1.25):

$-n$ is the total number of creators and annihilators in (1.13).

$-j_{1}, \cdots, j_{k}$ are the indices of the creators and annihilators in the 1-st Hilbert space (in the identification $\left.(1.6),(1.6)^{\prime}\right)$. These indices go with the operator $Q_{+}$ of (1.5).

- $j_{1}^{c}, \cdots, j_{n-k}^{c}$ as above, for the 2 -nd Hilbert space. These indices go with $Q_{-}$.

- Since the $j_{1}^{\iota}, \cdots, j_{n-k}^{\mathrm{c}}$ are uniquely determined by $j_{1}, \cdots, j_{k}$, summation is taken only over the latter indices.

$-x$ is the number of creators in the 1 -st space.

$-x^{\prime}$-as above for the 2 -nd space.

$-m$ is the number of creators in the 1-st space used to produce scalar products.

- $m^{\prime}$-as above for the 2 -nd space.

$-j_{t_{1}}, \cdots, j_{t_{1}}$ are the indices of the creators in the 1 -st space.

- $j_{i_{i}^{\prime}}^{\mathrm{c}}, \cdots, j_{l_{l^{\prime}}}^{\mathrm{c}}$-as above for the 2 -nd space.

$-j_{t_{1}}, \cdots, j_{\iota_{l,}}$ are the indices of the creators in the 1-st space used to produce scalar products.

- $j_{l_{, j}^{\prime}}^{\mathrm{c}}, \cdots, j_{l^{\prime}, w^{\prime}}^{\mathrm{c}}$-as above for the 2 -nd space.

- $\left(p_{1}, \cdots, p_{m}\right)$ (resp. $\left.\left(q_{1}, \cdots, q_{m}\right)\right)$ are refered to those creators (resp. annihilators) in the 1-st space used to produce scalar products of type II, i.e. with some $j_{q}-j_{p} \geq 2$.

- $\left(p_{1}^{\prime}, \cdots, p_{m m^{\prime}}^{\prime}\right)\left(\operatorname{resp} .\left(q_{1}^{\prime}, \cdots, q_{m^{\prime}}^{\prime}\right)\right)$-as above for the 2-nd space.

First of all, we estimate $\Delta_{n}^{(\lambda)}(1)$ defined in (1.22),

Lemma (1.1). There exists a constant $C$ satisfying

$$
\left|\Delta_{n}^{(\lambda)}(1)\right| \leq\|u\| \cdot\|v\| \cdot C^{n}(t \vee 1)^{n} \frac{1}{\left(\left[\frac{n}{2}\right]\right) !}
$$


Proof. Putting, in (1.22)

$$
s_{1}=\lambda^{2} t_{1}, \cdots, s_{n}=\lambda^{2} t_{n}
$$

one gets

$$
\begin{aligned}
& \left|\Delta_{n}^{(\lambda)}(1)\right| \leq \sum_{h=0}^{n} \sum_{1 \leq J_{1}<<J_{h} \leq n} \sum_{i=0}^{h} \sum_{m=0}^{n(h-\lambda)}
\end{aligned}
$$

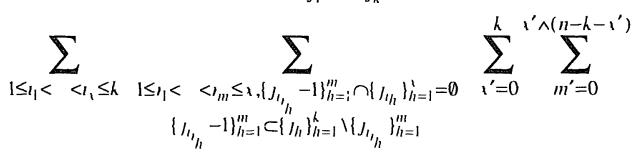

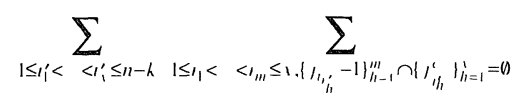

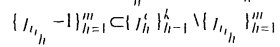

$$
\begin{aligned}
& \left|\left\langle u, D\left(j_{1}, \cdots, j_{h} ; i_{1}, \cdots, i_{1} ; i_{1}^{\prime}, \cdots, i_{1}^{\prime}\right) v\right\rangle\right| \\
& \int_{0}^{l} d s_{1} \int_{0}^{s_{1}} d s_{2} \cdots \int_{0}^{\varsigma_{n-1}} d s_{n}\left|F\left(s_{1}, \cdots, s_{n}, \lambda\right)\right|
\end{aligned}
$$

where, $F(\cdot, \ldots, \cdot, \lambda)$ has the form

$$
\begin{aligned}
& F\left(t_{1}, \cdots, t_{n} ; \lambda\right):=F_{1}\left(t_{l_{h_{1}}}, \cdots, t_{l_{l_{m}}} ; \lambda\right) \cdot F_{2}\left(t_{l_{i_{1}}}, \cdots, t_{l_{l_{i, m^{\prime}}}} ; \lambda\right):=
\end{aligned}
$$

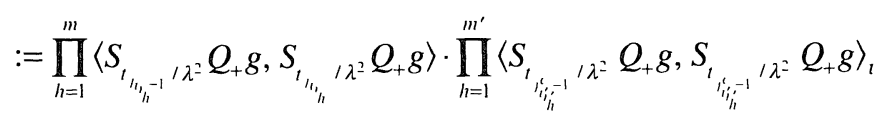

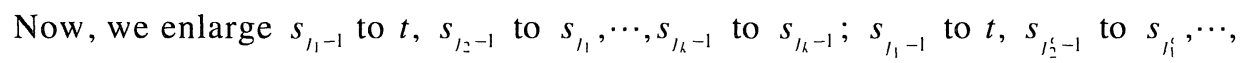
$s_{l_{n-k}-1}$ to $s_{l_{n-k-1}}$. Then since, by (1.10) and (1.14), the $j, j^{c}$ are a partition of $\{1, \cdots, n\}$, the integral term in (1.28) is dominated by

$$
\begin{gathered}
\int_{0}^{t} d s_{1} \int_{0}^{\prime_{1}} d s_{2} \cdots \int_{0}^{\prime_{h-1}} d s_{h}\left|F_{1}\left(s_{1}, \cdots, s_{h} ; \lambda\right)\right| \cdot \\
\int_{0}^{t} d s_{1}^{\prime} \int_{0}^{\prime_{1}^{\prime}} d s_{2}^{\prime} \cdots \int_{0}^{\prime_{n-h-1}^{\prime}} d s_{n-h}^{\prime}\left|F_{2}\left(s_{1}^{\prime}, \cdots, s_{n-h}^{\prime} ; \lambda\right)\right|
\end{gathered}
$$

Since each integral in (1.30) has the same form as the integral in (5.8b) in [4], we can apply to each of them the estimate (5.18) of [4] which leads to the following estimate (for some $C_{1}$ )

$$
\cdot\left|\Delta_{n}^{(\lambda)}(1)\right| \leq\|u\| \cdot\|v\| \cdot \sup _{0 \leq L \leq n} C_{1}^{n} \frac{(t \vee 1)^{n}}{\left(\left[\frac{k}{2}\right]\right) !\left(\left[\frac{n-k}{2}\right]\right) !} \leq\|u\| \cdot\|v\| \cdot C^{n} \frac{(t \vee 1)^{n}}{\left(\left[\frac{n}{2}\right]\right) !}
$$

With the same procedure, we get the uniform (in $\lambda$ ) estimate for $\Delta_{n}^{(\lambda)}(2)$ :

$$
\left|\Delta_{n}^{(\lambda)}(2)\right| \leq\|u\| \cdot\|v\| \cdot C^{n} \frac{(t \vee 1)^{n}}{[n / 3] !}
$$


which is the analogue of the estimate (5.34) of [4]. Summing up, we have the following

Lemma (1.2). There exists a constant $C$, such that, if $\Delta_{n}^{(\lambda)}(t)$ is defined by (1.19), then for each $n \in \mathbb{N}$

$$
\left|\Delta_{n}^{(\lambda)}(t)\right| \leq\|u\| \cdot\|v\| \cdot C^{n} \frac{(t \vee 1)^{n}}{\left(\left[\frac{n}{3}\right]\right) !}
$$

Now, we shall prove the analogue of Lemma (4.2) of [4].

Lemma (1.3)。For each $n \in \mathbb{N}, f_{1}, f_{2}, g \in K$,

$$
\lim _{\lambda \rightarrow 0} \Delta_{n}^{(\lambda)}(2)=0
$$

Proof. The proof is an easy modification of the proof of Lemma (4.2) in [4] and we only sketch the main idea of it. Consider, for example, $[I \otimes I I]$. The general term of this type will be majorized in modulus by

$$
\begin{gathered}
\mid\left\langle W\left(\lambda \int_{S_{1} / \lambda^{2}}^{T_{1} / \lambda^{2}} S_{u} Q_{+} f_{1} d u\right) \Phi_{F} \otimes W\left(\lambda \int_{S_{1} / \lambda^{2}}^{T_{1} / \lambda^{2}} S_{u} Q_{-} f_{1} d u\right) \Phi_{F}^{t},\right. \\
\int_{0}^{t} d t_{1} \int_{0}^{t_{1}} d t_{2} \cdots \int_{0}^{t_{n-1}} d t_{n}\left|I_{v}^{\varepsilon(1)} \otimes I I_{v}^{\varepsilon\left(l^{\prime}\right)}\right| \\
\left.W\left(\lambda \int_{S_{2} / \lambda^{2}}^{T_{2} / \lambda^{2}} S_{u} Q_{+} f_{2} d u\right) \Phi_{F} \otimes W\left(\lambda \int_{S_{2} / \lambda^{2}}^{T_{2} / \lambda^{2}} S_{u} Q_{-} f_{2} d u\right) \Phi_{F}^{l}\right\rangle \mid
\end{gathered}
$$

With the same trick as in the proof of Lemma (1.1), we majorize (1.35) with a product of two factors of the form (1.30). We then use the estimate (1.26) to majorize the first term of this product and we obtain that (1.35) is less or equal than

$$
C^{h} \frac{(t \vee 1)^{h}}{[k / 2] !} \int_{0}^{t} d s_{1}^{\prime} \int_{0}^{\prime_{1}^{\prime}} d s_{2}^{\prime} \cdots \int_{0}^{\prime_{n-h-1}^{\prime}} d s_{n-h}^{\prime} F_{2}\left(s_{1}^{\prime}, \cdots, s_{n-h}^{\prime} ; \lambda\right)
$$

But, the integral on the right hand side of (1.36) is of the same form as the right hand side of (4.16) of [4] which goes to zero because of Lemma (4.2) of [4]. The argument for the other two types of terms is similar.

The following is the analogue of Theorem (5.1) of [4].

Lemma (且。4)。For each $n \in \mathbb{N}, f_{1}, f_{2}, g \in K, t \geq 0$

$$
\lim _{\lambda \rightarrow 0} \Delta_{n}^{(\lambda)}(1)=
$$




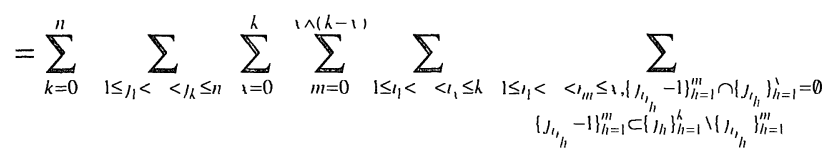

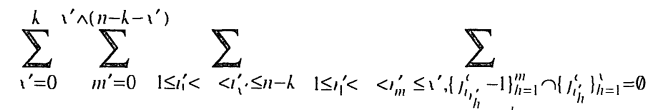

$$
\begin{aligned}
& \left\{h_{i}^{i},-1\right\}_{h=1}^{m} \subset\left\{\mid J_{h}^{i}\right\}_{h=1}^{n-k} \backslash\left\langle J_{i}^{i} i_{h}^{m}\right\}_{h=1}^{m} \\
& \left\langle u, D_{n}\left(j_{1}, \cdots, j_{k} ; i_{1}, \cdots, i_{1} ; i_{1}^{\prime}, \cdots, i_{1_{1}^{\prime}}^{\prime}\right) v\right\rangle
\end{aligned}
$$

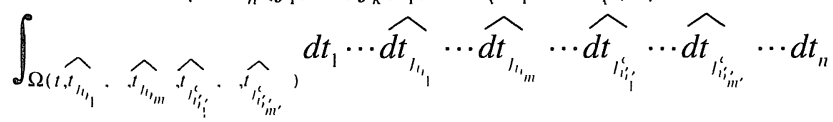

$$
\begin{aligned}
& \left(Q_{+} g \mid Q_{+} g\right)_{-}^{m} \cdot\left(Q_{+} f_{1} \mid Q_{+} g\right)^{1-m} \cdot\left(Q_{+} g \mid Q_{+} f_{2}\right)^{k-1-m}
\end{aligned}
$$

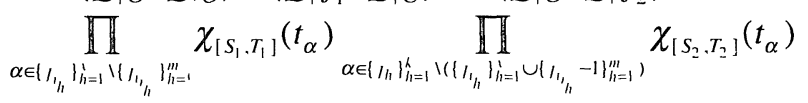

$$
\begin{aligned}
& \left(Q^{-} g \mid Q^{-} g\right)_{+}^{m m^{\prime}} \cdot\left(Q^{-} g \mid Q^{-} f_{1}\right)^{1^{\prime}-m^{\prime}} \cdot\left(Q^{-} f_{2} \mid Q^{-} g\right)^{n-h-1^{\prime}-m^{\prime}}
\end{aligned}
$$

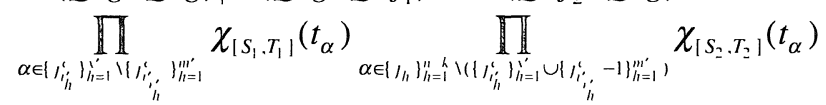

$$
\begin{aligned}
& \left\langle W\left(\chi_{\left[S_{1}, T_{1}\right]} \otimes Q_{+} f_{1}\right) \Psi_{F}, W\left(\chi_{\left[S_{2}, T_{2}\right]} \otimes Q_{+} f_{2}\right) \Psi_{F}\right\rangle \\
& \left\langle W\left(\chi_{\left[S_{2}, T_{2}\right]} \otimes Q^{-} f_{2}\right) \Psi_{F}^{l}, W\left(\chi_{\left[S_{1}, T_{1}\right]} \otimes Q^{-} f_{1}\right) \Psi_{F}^{l}\right\rangle
\end{aligned}
$$

where, $\left(Q^{-} f \mid Q^{-} g\right)_{+},\left(Q^{+} f \mid Q^{+} g\right)_{-}$are defined by (1.39a) below,

$$
Q^{-}:=\sqrt{\frac{Q-1}{2}}=\iota^{-1}\left(Q_{-}\right)
$$

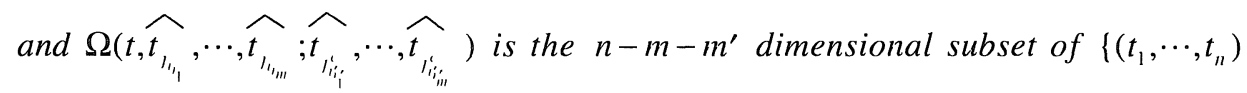
$\left.\in \mathbb{R}^{n} ; 0 \leq t_{n} \leq \cdots \leq t_{1} \leq t\right\}$ in which, the variables

$$
t_{l_{t_{1}}}, \cdots, t_{l_{t_{t+1}}} ; t_{l_{i_{1}}^{t_{1}}}, \cdots, t_{l_{i_{i, i}}}
$$

are suppressed.

Proof. Also in this case we limit ourselves to a sketch of the proof, which is an easy adaptation of that of Theorem (5.1) in [4]. By inspection of the expression for $\Delta_{n}^{(\lambda)}(1)$ and using again the fact that the $\left(j, j^{c}\right)$-indices are a partition of $\{1, \cdots, n\}$, we see that we can apply separately to the $\left(t_{1-1}-t_{1}\right)$ variables and the $\left(t_{t^{\prime}-1}-t_{t^{\prime}}\right)$-variables, the argument of the second part of the proof of Theorem (5.1) in [4]. Recalling that the indices $j_{l_{1}}, j_{l^{\prime}}$, here play the role of the indices $j$, there (i.e. they label the factors $A_{t_{x},} A_{t_{x}}^{+}$) and that the change of variables needed involve only the indices $j_{t_{1}-1}, j_{t^{\prime}} ; j_{t_{i}^{\prime}-1}^{c}, j_{t^{\prime}}^{c}$. We see that the missing variables in the integral

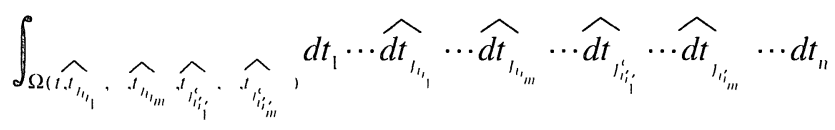


(i.e. the $\widehat{t_{\alpha}}$ ) correspond to the factors

$$
\left(Q_{+} g \mid Q_{+} g\right)_{-}=\int_{-\infty}^{0} d t\left\langle Q_{+} g, S_{t} Q_{+} g\right\rangle,\left(Q_{-} g \mid Q_{-} g\right)_{+} \int_{0}^{+\infty} d t\left\langle Q_{+} g, S_{t} Q_{+} g\right\rangle
$$

The apperance of $Q^{-}$is then justified by the identity:

$$
\left(Q_{-} f \mid Q_{-} g\right)_{1}=\left(Q^{-} g \mid Q^{-} f\right) ; f, g \in K
$$

which easily follows from (1.38) and the definition of $(\cdot \mid \cdot)$.

Putting together Lemma (1.1), Lemma (1.2), Lemma (1.3) and Lemma (1.4), one has

Lemma (1.5). In the above notations and assumptions, one has, for each $f_{1}, f_{2}, g \in K, T_{1}, T_{2}, S_{1}, S_{2} \in \mathbb{R}$ and $t \geq 0$, the limit

$$
\lim _{\lambda \rightarrow 0}\left\langle u \otimes W_{Q}\left(\lambda \int_{S_{1} / \lambda^{2}}^{T_{1} / \lambda^{2}} S_{u} f_{1} d u\right) \Phi_{Q}, U^{(\lambda)}\left(t / \lambda^{2}\right) \nu \otimes W_{Q}\left(\lambda \int_{S_{2} / \lambda^{2}}^{T_{2} / \lambda^{2}} S_{u} f_{2} d u\right) \Phi_{Q}\right\rangle
$$

exists and is equal to

$$
\begin{aligned}
& \sum_{n=0}^{\infty} \sum_{h=0}^{n} \sum_{1 \leq J_{1}<<l_{k} \leq n} \sum_{i=0}^{h} \sum_{m=0}^{n(k-1)} \sum_{1 \leq 1_{1}<<l_{1} \leq k}
\end{aligned}
$$

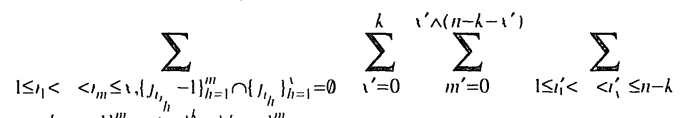

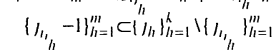

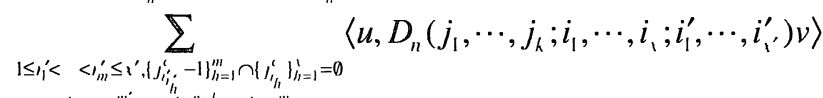

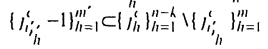

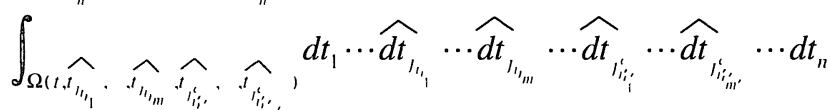

$$
\begin{aligned}
& \left(Q_{+} g \mid Q_{+} g\right)_{-}^{m} \cdot\left(Q_{+} f_{1} \mid Q_{+} g\right)^{\imath-m} \cdot\left(Q_{+} g \mid Q_{+} f_{2}\right)^{h-\imath-m}
\end{aligned}
$$

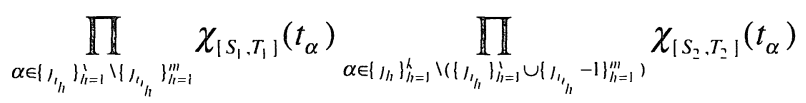

$$
\begin{aligned}
& \left(Q^{-} g \mid Q^{-} g\right)_{+}^{m^{\prime}} \cdot\left(Q^{-} g \mid Q^{-} f_{1}\right)^{\prime^{\prime}-m^{\prime}} \cdot\left(Q^{-} f_{2} \mid Q^{-} g\right)^{n-h-1^{\prime}-m^{\prime}}
\end{aligned}
$$

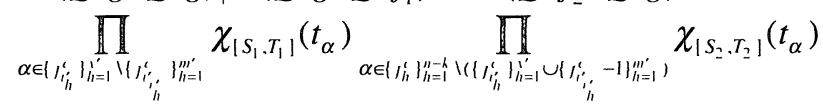

$$
\begin{aligned}
& \left\langle W\left(\chi_{\left[S_{1}, T_{1}\right]} \otimes Q_{+} f_{1}\right) \Psi_{F}, W\left(\chi_{\left[S_{2}, T_{2}\right]} \otimes Q_{+} f_{2}\right) \Psi_{F}\right\rangle \\
& \left\langle W\left(\chi_{\left.\mid S_{2}, T_{2}\right]} \otimes Q^{-} f_{2}\right) \Psi_{F}^{\prime}, W\left(\chi_{\left[S_{1}, T_{1}\right]} \otimes Q^{-} f_{1}\right) \Psi_{F}^{\prime}\right\rangle
\end{aligned}
$$

Remark. In [4] we repeatedly stressed that the iterated series converges weakly on the domain of collective coherent vectors. The same type of convergence is assured in the present case as used in Lemma (1.5). 
Denoting by $G(u, t)$ the limit (1.40), in the following Lemma, we shall compute $\frac{d}{d t} G(u, t)$.

Lemma (1.6). For each $f_{1}, f_{2}, g \in K, T_{1}, T_{2}, S_{1}, S_{2} \in \mathbf{R}$, and $t \geq 0, G(u, t)$ is a.e. differentiable for $t>0$ and

$$
\begin{gathered}
G(u, t)=G(u, 0)+ \\
\int_{0}^{t} d s\left(\left(Q_{+} f_{1} \mid Q_{+} g\right) G\left(D^{+} u, s\right) \chi_{\left[S_{1}, T_{1}\right]}(s)+\left(Q_{+} g \mid Q_{+} f_{2}\right) G(-D u, s) \chi_{\left[s_{2}, T_{2}\right]}(s)\right. \\
+\left(Q^{-} g \mid Q^{-} f_{1}\right) G(-D u, s) \chi_{\left[S_{1}, T_{1}\right]}(s)+\left(Q^{-} f_{2} \mid Q^{-} g\right) G\left(D^{+} u, s\right) \chi_{\left[S_{2}, T_{2}\right]}(s) \\
\left.+\left(Q_{+} g \mid Q_{+} g\right)_{-} G\left(-D^{+} D u, s\right)+\left(Q^{-} g \mid Q^{-} g\right)_{+} G\left(-D D^{+} u, s\right)\right)
\end{gathered}
$$

Proof. First of all, notice that the limit (1.40) exists, is sesquilinear in $u, v$ and is dominated by $\|u\| \cdot\|v\|$. Hence there exists a contraction $V_{t}:=V_{t}\left(f_{1}, f_{2}, S_{1}, S_{2}, T_{1}, T_{2}\right): H_{0} \rightarrow H_{0}$ such that the limit of the left hand side of (1.40) is equal to

$$
\langle u, V, v\rangle=: G(u, t)
$$

Moreover, from Lemma (1.1), Lemma (1.2), Lemma (1.3) and Lemma (1.4), $G(u, t)$ is a.e. differentiable for $t>0$.

In order to deduce an equation for $G(u, t)$, first notice that, for fixed $\lambda$, one has:

$$
\begin{gathered}
\frac{d}{d t}\left\langle u \otimes W_{Q}\left(\lambda \int_{S_{1} / \lambda^{2}}^{T_{1} / \lambda^{-}} S_{u} f_{1} d u\right) \Phi_{Q}, U^{(\lambda)}\left(t / \lambda^{2}\right) v \otimes W_{Q}\left(\lambda \int_{S_{2} / \lambda^{2}}^{T_{2} / \lambda^{2}} S_{u} f_{2} d u\right) \Phi_{Q}\right\rangle \\
=\frac{d}{d t}\left\langle u \otimes W_{Q}\left(\lambda \int_{S_{1} / \lambda^{2}}^{T_{1} / \lambda^{2}} S_{u} f_{1} d u\right) \Phi_{Q}, \sum_{n=1}^{\infty}(-i)^{n} \lambda^{n} \int_{0}^{t / \lambda^{2}} d t_{1} \int_{0}^{t_{1}} d t_{2} \cdots \int_{0}^{t_{n-1}} d t_{n}\right. \\
\left.V_{g}\left(t_{1}\right) \cdots V_{g}\left(t_{n}\right) \cdot v \otimes W_{Q}\left(\lambda \int_{S_{2} / \lambda^{2}}^{T_{2} / \lambda^{2}} S_{u} f_{2} d u\right) \Phi_{Q}\right\rangle \\
=\left\langle u \otimes W_{Q} \lambda \int_{S_{1} / \lambda^{2}}^{T_{1} / \lambda^{2}} S_{u} f_{1} d u\right) \Phi_{Q},(-i) \frac{1}{\lambda} V_{u}\left(t / \lambda^{2}\right) \\
=\left\langle u \otimes W_{Q}\left(\lambda \int_{S_{1} / \lambda^{2}}^{T_{1} / \lambda^{2}} S_{u} f_{1} d u\right) \Phi_{Q},-\frac{1}{\lambda}\left[-D \otimes A_{Q}^{+}\left(S_{t / \lambda^{2}} g\right)+D^{+} \otimes A_{Q}\left(S_{t / \lambda^{2}} g\right)\right]\right. \\
\left.\cdot U^{(\lambda)}\left(t / \lambda^{2}\right) v \otimes W_{Q}\left(\lambda \int_{S_{2} / \lambda^{2}}^{T_{2} / \lambda^{2}} S_{u} f_{2} d u\right) \Phi_{Q}\right\rangle \\
\left.=\left\langle u \otimes W_{S_{2} / \lambda^{2}}^{T_{2} / \lambda^{2}} S_{u} f_{2} d u\right) \Phi_{Q}\right\rangle \\
-\frac{1}{\lambda}\left[-D \int_{S_{1} / \lambda^{2}}^{T_{1} / \lambda^{2}} S_{u} Q_{+} f_{1} d u\right) \Phi_{F} \otimes W\left(\lambda \int_{S_{1} / \lambda^{2}}^{T_{1} / \lambda^{2}} S_{u} Q_{-} f_{1} d u\right) \Phi_{F}^{t}, \\
\left.A^{+}\left(S_{t / \lambda^{2}} Q_{+} g\right) \otimes 1+1 \otimes A\left(S_{t / \lambda^{2}} Q_{-} g\right)\right)
\end{gathered}
$$




$$
\begin{gathered}
\left.+D^{+} \otimes\left(A\left(S_{t / \lambda^{2}} Q_{+} g\right) \otimes 1+1 \otimes A^{+}\left(S_{t / \lambda^{2}} Q_{-} g\right)\right)\right] \\
\left.\cdot U^{(\lambda)}\left(t / \lambda^{2}\right) v \otimes W\left(\lambda \int_{S_{2} / \lambda^{2}}^{T_{2} / \lambda^{2}} S_{u} Q_{+} f_{2} d u\right) \Phi_{F} \otimes W\left(\lambda \int_{S_{2} / \lambda^{2}}^{T_{2} / \lambda^{2}} S_{u} Q_{-} f_{2} d u\right) \Phi_{F}^{2}\right\rangle \\
=\left\langle u \otimes W\left(\lambda \int_{S_{1} / \lambda^{2}}^{T_{1} / \lambda^{2}} S_{u} Q_{+} f_{1} d u\right) \Phi_{F} \otimes W\left(\lambda \int_{S_{1} / \lambda^{2}}^{T_{1} / \lambda^{2}} S_{u} Q_{-} f_{1} d u\right) \Phi_{F}^{l},\right. \\
-\frac{1}{\lambda}\left[-D \otimes A^{+}\left(S_{t / \lambda^{2}} Q_{+} g\right) \otimes 1+D^{+} \otimes A\left(S_{t / \lambda^{2}} Q_{+} g\right) \otimes 1\right] . \\
\left.\cdot U^{(\lambda)}\left(t / \lambda^{2}\right) v \otimes W\left(\lambda \int_{S_{2} / \lambda^{2}}^{T_{2} / \lambda^{2}} S_{u} Q_{+} f_{2} d u\right) \Phi_{F} \otimes W\left(\lambda \int_{S_{2} / \lambda^{2}}^{T_{2} / \lambda^{2}} S_{u} Q_{-} f_{2} d u\right) \Phi_{F}^{l}\right\rangle \\
+\left\langle u \otimes W\left(\lambda \int_{S_{1} / \lambda^{2}}^{T_{1} / \lambda^{2}} S_{u} Q_{+} f_{1} d u\right) \Phi_{F} \otimes W\left(\lambda \int_{S_{1} / \lambda^{2}}^{T_{1} / \lambda^{2}} S_{u} Q_{-} f_{1} d u\right) \Phi_{F}^{l},\right. \\
\left.-\frac{1}{\lambda}\left[-D \otimes 1 \otimes A\left(S_{t / \lambda^{2}} Q_{-} g\right)\right)+D^{+} \otimes 1 \otimes A^{+}\left(S_{t / \lambda^{2}} Q_{-} g\right)\right] \\
\left.\cdot U^{(\lambda)}\left(t / \lambda^{2}\right) v \otimes W\left(\lambda \int_{S_{2} / \lambda^{2}}^{T_{2} / \lambda^{2}} S_{u} Q_{+} f_{2} d u\right) \Phi_{F} \otimes W\left(\lambda \int_{S_{2} / \lambda^{2}}^{T_{2} / \lambda^{2}} S_{u} Q_{-} f_{2} d u\right) \Phi_{F}^{l}\right\rangle \text { a.e. }
\end{gathered}
$$

Notice that the right hand side of (1.42) can be written in the form $I_{\lambda}+I I_{\lambda}$, where, $I_{\lambda}$ and $I I_{\lambda}$ are the analogues of the quantities (6.8) and (6.9) in [4]. So, the results of the Lemmas (6.2), (6.3) and (6.4) of [4] can be applied to these quantities with only verbal changes. From this, we get (1.41).

In the following we shall denote $\left\{\mathscr{H}_{1 \otimes Q}, \pi_{1 \otimes Q}, \Psi_{1 \otimes Q}\right\}$ the gauge invariant quasi free Gaussian representation of the $\mathrm{CCR}$ on $L^{2}(\mathbb{R}, d t, K)$ with mean zero and covariance $1 \otimes Q$ and $A\left(\chi_{[0, t]} \otimes g\right), A^{+}\left(\chi_{\mid 0, t]} \otimes g\right)$ the associated $Q$-Brownian Motion on $L^{2}\left(\mathbb{R}_{+}, d t ; K\right)$ in the sense of the definition (2.3) of [4].

Theorem (1.7). In the above notations and assumptions, for each $f_{1}, f_{2}$, $g \in K, T_{1}, T_{2}, S_{1}, S_{2} \in \mathbb{R}, t \geq 0$ and $u, v \in H_{0}, D \in B\left(H_{0}\right)$,

$$
\begin{gathered}
\lim _{\lambda \rightarrow 0}\left\langle u \otimes W_{Q}\left(\lambda \int_{S_{1} / \lambda^{2}}^{T_{1} / \lambda^{2}} S_{u} f_{1} d u\right) \Phi_{Q}, U^{(\lambda)}\left(t / \lambda^{2}\right) v \otimes W_{Q}\left(\lambda \int_{S_{2} / \lambda^{2}}^{T_{2} / \lambda^{2}} S_{u} f_{2} d u\right) \Phi_{Q}\right\rangle \\
=\left\langle u \otimes W_{1 \otimes Q}\left(\chi_{\left|S_{1} \cdot T_{1}\right|} \otimes f_{1}\right) \Psi_{1 \otimes Q}, U(t) v \otimes W_{1 \otimes Q}\left(\chi_{\left|S_{2} \cdot T_{2}\right|} \otimes f_{2}\right) \Psi_{1 \otimes Q}\right\rangle
\end{gathered}
$$

where,

$$
\left\langle\Psi_{1 \otimes Q}, W_{1 \otimes Q}(\xi \otimes f) \Psi_{1 \otimes Q}\right\rangle=\exp \left(-\frac{1}{2}\langle\xi \otimes f,(1 \otimes Q)(\xi \otimes f)\rangle\right)
$$

for $\xi \in L^{2}(\mathbb{R})$, and $U(t)$ is the unique solution of quantum stochastic differential equation

$$
\begin{gathered}
U(t)=1+\int_{0}^{t}\left(D \otimes d A_{Q}^{+}(s, g)-D^{+} \otimes d A_{Q}(s, g)+\right. \\
\left.+\left(-\left(Q_{+} g \mid Q_{+} g\right)_{-} D^{+} D \otimes 1-\left(Q^{-} g \mid Q^{-} g\right)_{+} D D^{+} \otimes 1\right) d s\right) U(s)
\end{gathered}
$$

on 


$$
H_{0} \otimes \mathscr{H}_{1 \otimes Q} \cong H_{0} \otimes \Gamma\left(L^{2}(\mathbf{R}) \otimes(K,(\cdot \mid \cdot))\right) \otimes \Gamma\left(L^{2}(\mathbf{R}) \otimes\left(K_{1},(\cdot \mid \cdot)_{1}\right)\right)
$$

with

$$
Q_{+}=\sqrt{\frac{Q+1}{2}} ; Q^{-}=\sqrt{\frac{Q-1}{2}}
$$

Moreover $U(t)$ is unitary for each $t \geq 0$.

Proof. For each $u \in H_{0}$, put

$$
F(u, t)=\left\langle u \otimes W_{1 \otimes Q}\left(\chi_{\left[S_{1}, T_{1}\right]} \otimes f_{1}\right) \Psi_{1 \otimes Q}, U(t) v \otimes W_{1 \otimes Q}\left(\chi_{\left[S_{2}, T_{2}\right]} \otimes f_{2}\right) \Psi_{1 \otimes Q}\right\rangle
$$

It is easy to show $F(u, t)$ satisfies (1.41). Since we know from [8] that the equation (1.45) has a unique solution, it follows that $F(u, t)$ is the unique solution of (1.41). So, $F(u, t)=G(u, t)$ for all $t \geq 0$.

To prove unitarity, first notice that from (1.44) one easily deduces the covariance of the quantum BM $A_{Q}(t, g), A_{Q}^{+}(t, g)$ :

$$
\begin{gathered}
\left\langle\psi_{1 \otimes Q}, A_{Q}^{+}(\alpha \otimes f) A_{Q}(\beta \otimes g) \psi_{1 \otimes Q}\right\rangle \\
=\left\langle\beta \otimes g, \frac{1 \otimes Q-1 \otimes 1}{2} \alpha \otimes f\right\rangle=\langle\beta, \alpha\rangle\left(g \mid \frac{Q-1}{2} f\right) \\
\left\langle\psi_{1 \otimes Q}, A_{Q}(\alpha \otimes f) A_{Q}^{+}(\beta \otimes g) \psi_{1 \otimes Q}\right\rangle \\
=\left\langle\alpha \otimes f, \frac{1 \otimes Q-1 \otimes 1}{2} \beta \otimes g\right\rangle=\langle\alpha, \beta\rangle\left(f \mid \frac{Q+1}{2} g\right)
\end{gathered}
$$

This implies that the Itô table is:

$$
\begin{gathered}
d A_{Q}(t, f) \quad d A_{Q}^{+}(t, g)=\left\langle f, \frac{Q+1}{2} g\right\rangle d t \\
d A_{Q}^{+}(t, f) d A_{Q}(t, g)\left\langle g, \frac{Q-1}{2} f\right\rangle d t \\
d A d A=d A^{+} d A^{+}=0
\end{gathered}
$$

Moreover since $(f \mid f)_{-}={\overline{(f \mid f)_{+}}}_{\text {it follows from a simple calculation that }}$

$$
\begin{aligned}
& \left(Q_{+} g \mid Q_{+} g\right)_{-}=\frac{1}{2}\left(g \mid \frac{Q+1}{2} g\right)+i \operatorname{Im}\left(g \mid \frac{Q+1}{2} g\right)_{-} \\
& \left(Q_{-} g \mid Q_{-} g\right)_{+}=\frac{1}{2}\left(g \mid \frac{Q-1}{2} g\right)+i \operatorname{Im}\left(g \mid \frac{Q-1}{2} g_{+}\right)
\end{aligned}
$$

the equation (1.45) could be rewritten in the form

$$
\begin{gathered}
U(t)=1+\int_{0}^{t}\left[D \otimes d A_{Q}^{+}(s, g)-D^{+} \otimes d A_{Q}(s, g)\right. \\
-\frac{1}{2} D^{+} D\left(Q_{+} g \mid Q_{+} g\right) d s-\frac{1}{2} D D^{+}\left(Q_{-} g \mid Q_{-} g\right) d s \\
\left.+i\left(-D^{+} D \operatorname{Im}\left(Q_{+} g \mid Q_{+} g\right)_{-}-D D^{+} \operatorname{Im}\left(Q_{-} g \mid Q_{-} g\right)_{+}\right) d s\right] U(s)
\end{gathered}
$$


which, in view of the Ito table (1.48), (1.49), (1.50) and of the identities (1.51), (1.52) makes evident that the formal unitary condition of [11] and [12] (in [8] only the Fock case, not the general quasi-free case, as needed here, is considered) is satisfied.

Since $D$ is bounded, the formal unitarity condition guarantees existence, uniqueness and unitarity [12].

\section{§2. The Uniform Estimates of the Heisenberg Evolution: Fock Case}

In the following sections, we shall deal with the Langevin case, that is, we shall consider the weak coupling limit of the matrix elements in the collective coherent vectors of $U^{(\lambda)}(t)(X \otimes 1) U^{(\lambda)+}(t)$ and their properties. For $X \in B\left(H_{0}\right)$, $t \geq 0$, put

$$
X^{(\lambda)}(t)=U^{(\lambda)}(t)(X \otimes 1) U^{(\lambda)+}(t)
$$

then,

$$
\begin{gathered}
\frac{d}{d t} X^{(\lambda)}(t)=\left(\frac{d}{d t} U^{(\lambda)}(t)\right)(X \otimes 1) U^{(\lambda)+}(t)+U^{(\lambda)}(t)(X \otimes 1)\left(\frac{d}{d t} U^{(\lambda)+}(t)\right) \\
=\frac{1}{i} \lambda V(t) X^{(\lambda)}(t)-\frac{1}{i} \lambda X^{(\lambda)} V^{+}(t)=\frac{1}{i} \lambda\left[V(t), X^{(\lambda)}(t)\right]
\end{gathered}
$$

and

$$
X^{(\lambda)}(0)=X \otimes 1
$$

So,

$$
\begin{gathered}
X^{(\lambda)}(t)=X \otimes 1+\sum_{n=1}^{\infty}(-i)^{n} \lambda^{n} \int_{0}^{t_{1}} d t_{1} \int_{0}^{t_{1}} d t_{2} \cdots \int_{0}^{t_{n-1}} d t_{n} \\
{\left[V\left(t_{1}\right),\left[V\left(t_{2}\right), \cdots,\left[V\left(t_{n}\right), X \otimes 1\right] \cdots\right]\right]}
\end{gathered}
$$

If $n \in \mathbb{N}$, and $B, A_{1}, \cdots, A_{n}$, are operators, there exists a subset.$_{n}{ }^{0}$ of the $n$ permutations $S_{n}$ such that

$$
\left[A_{1}, \cdots,\left[A_{n}, B\right] \cdots\right]=\sum_{d=0}^{n} \sum_{\sigma \in /, n} \theta(\sigma) A_{\sigma(1)} \cdots A_{\sigma(d)} B A_{\sigma(d+1)} \cdots A_{\sigma(n)}
$$

where $\theta(\sigma)= \pm 1$ and is determined uniquely by $\sigma$, moreover

$$
\left|y_{n}{ }^{\prime}\right|=2^{n}
$$

So, for each $n \in \mathbf{N}$, one has 
So, for each $n \in \mathbf{N}$, one has

$$
\begin{aligned}
& \left.\left[V\left(t_{1}\right), V\left(t_{2}\right), \cdots,\left[V\left(t_{n}\right), X \otimes 1\right] \cdots\right]\right]=\sum_{d=0}^{n} \sum_{\sigma \in /, n} \theta(\sigma) V\left(t_{\sigma(1)}\right) \cdots V\left(t_{\sigma(d)}\right) \\
& (X \otimes 1) V\left(t_{\sigma(d+1)}\right) \cdots V\left(t_{\sigma(n)}\right) \\
& =\sum_{d=0}^{n} \sum_{\sigma \in S_{n}^{\prime}} \sum_{\varepsilon \in\{0.1)^{n}} \theta(\sigma) D_{\varepsilon(1)} \cdots D_{\varepsilon(d)} X D_{\varepsilon(d+1)} \cdots D_{\varepsilon(n)} \otimes i^{n} A^{\varepsilon(1)}\left(S_{\left.t_{\sigma(1, !}\right)}\right) \cdots A^{\varepsilon(n)}\left(S_{\left.t_{(I n, k}\right)}\right)
\end{aligned}
$$

Lemma (2.1). There is a constant $C$, for each $n \in \mathbf{N}, f_{1}, f_{2}, g \in \mathscr{K}, u, v$ $\in H_{0}, X, D \in B\left(H_{0}\right), T_{1}, S_{1}, T_{2}, S_{2} \in \mathbf{R}, t \geq 0$

$$
\begin{gathered}
\mid\left\langle u \otimes \Phi\left(\lambda \int_{S_{1} / \lambda^{2}}^{T_{1} / \lambda^{2}} S_{u} f_{1} d u\right), \lambda^{n} \int_{0}^{t / \lambda^{2}} d t_{1} \int_{0}^{t_{1}} d t_{2} \cdots \int_{0}^{t_{n-1}} d t_{n}\right. \\
\left.\left[V\left(t_{1}\right),\left[V\left(t_{2}\right), \cdots,\left[V\left(t_{n}\right), X \otimes 1\right] \cdots\right]\right] v \otimes \Phi\left(\lambda \int_{S_{2} / \lambda^{2}}^{T_{2} / \lambda^{2}} S_{u} f_{2} d u\right)\right\rangle \mid \\
\leq\|u\| \cdot\|v\| \cdot C^{n} \frac{(t \vee 1)^{n}}{\left(\left[\frac{n}{3}\right]\right) !}
\end{gathered}
$$

Proof. In (2.6), for each $\sigma \in \mathscr{Y}_{n}^{0}$ (introduced after formula (2.4)), in analogy with Lemma (4.1) of [4], the product

$$
A^{\varepsilon(1)}\left(S_{t_{\sigma 11}} g\right) \cdots A^{\varepsilon(n)}\left(S_{t_{\sigma(n}} g\right)
$$

can be written as the sum of two terms: the collective term $I_{q}^{\varepsilon}(\sigma)$ and the negligible term $I I_{g}^{\varepsilon}(\sigma)$ with:

$$
\begin{aligned}
& I_{q}^{\varepsilon}(\sigma)=\sum_{m=0}^{h \wedge(n-h)} \sum_{\substack{\left.\left.1 \leq q_{1}<<q_{m} \leq n,\left(\mid q_{h}, p_{h}\right\}_{h=1}^{m}, \sigma, 1\right) \\
\left.\left\{q_{1}\right\}_{h=1}^{\prime \prime} \subseteq\right|_{h},\right\}_{h=1}^{m}}}^{\prime}
\end{aligned}
$$

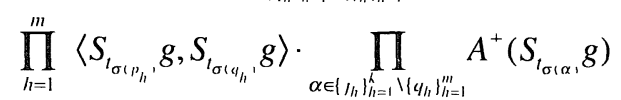

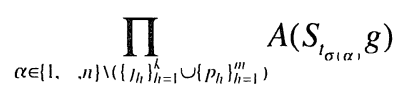

and

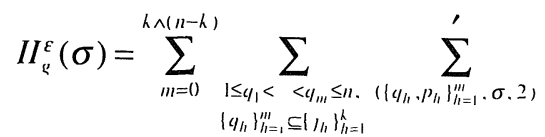

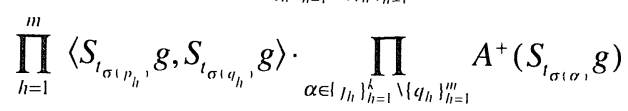

$$
\begin{aligned}
& \prod_{\alpha \in\left\{1 . . n \backslash \backslash\left(\{/ h\}_{h=1}^{\infty} \cup\left\{p_{n}\right\}\right\}_{h=1}^{m}\right)} A\left(S_{l_{\sigma \prime \sigma}} g\right)
\end{aligned}
$$


where, here and in the following, $\sum_{\left(\left\{q_{h}, p_{h}\right\}_{h=1}^{\prime m} \sigma .1\right)}$ means the sum for all $\left\{p_{h}\right\}_{h=1}^{m} \subseteq$ $\{1, \cdots, n\} \backslash\left\{j_{h}\right\}_{h=1}^{h},\left|\left\{p_{h}\right\}_{h=1}^{m}\right|=m ; p_{h}<q_{h},\left|\sigma\left(q_{h}\right)-\sigma\left(p_{h}\right)\right|=1$, for all $h=1, \cdots, m$, and $\sum_{\left\{\left\{q_{h}, p_{h}\right\}_{h=1}^{\prime \prime}, \sigma .2\right)}^{\prime}$ means the sum for all $\left\{p_{h l}\right\}_{h=1}^{m} \subset\{1, \cdots, n\} \backslash\left\{j_{h}\right\}_{h=1}^{k} ; p_{h}<q_{h}$ for $h=1$, $\cdots, m,\left|\left\{p_{h}\right\}_{h=1}^{m}\right|=m,\left|\sigma\left(q_{h}\right)-\sigma\left(p_{h}\right)\right| \geq 2$ for some $h=1, \cdots, m$.

Using Lemmas (1.1) and (1.2) above, for each $d=0,1, \cdots, n$, we easily get the estimates:

$$
\begin{gathered}
\mid\left\langle u \otimes \Phi\left(\lambda \int_{S_{1} / \lambda^{2}}^{T_{1} / \lambda^{2}} S_{u} f_{1} d u\right), \lambda^{n} \int_{0}^{t / \lambda^{2}} d t_{1} \int_{0}^{t_{1}} d t_{2} \cdots \int_{0}^{t_{n-1}} d t_{n}\right. \\
\left.\sum_{\varepsilon \in\{0.1\}^{n} \varepsilon \in\{0.1\}^{n}} D_{\varepsilon(1)} \cdots D_{\varepsilon(d)} X D_{\varepsilon(d+1)} \cdots D_{\varepsilon(n)} \otimes i^{n} I_{g}^{\varepsilon}(\sigma) v \otimes \Phi\left(\lambda \int_{S_{2} / \lambda^{2}}^{T_{2} / \lambda^{2}} S_{u} f_{2} d u\right)\right\rangle \mid \\
\leq\|u\| \cdot\|v\| \cdot C_{1}^{n} \frac{(t \vee 1)^{n}}{\left(\left[\frac{n}{2}\right]\right) !}
\end{gathered}
$$

and

$$
\begin{gathered}
\mid\left\langle u \otimes \Phi\left(\lambda \int_{S_{1} / \lambda^{2}}^{T_{1} / \lambda^{2}} S_{u} f_{1} d u\right), \lambda^{n} \int_{0}^{t / \lambda^{2}} d t_{1} \int_{0}^{t_{1}} d t_{2} \cdots \int_{0}^{t_{n-1}} d t_{n}\right. \\
\sum_{\varepsilon \in\{0.1)^{n}} \sum_{\varepsilon \in\{0.1\}^{n}} D_{\varepsilon(1)} \cdots D_{\varepsilon(d)} X D_{\varepsilon(d+1)} \cdots D_{\varepsilon(n)} \otimes i^{n} I I_{g}^{\varepsilon}(\sigma) \\
\left.\nu \otimes \Phi\left(\lambda \int_{S_{2} / \lambda^{2}}^{T_{2} / \lambda^{2}} S_{u} f_{2} d u\right)\right\rangle \mid \leq\|u\| \cdot\|v\| \cdot C_{2}^{n} \frac{(t \vee 1)^{n}}{\left(\left[\frac{n}{3}\right]\right) !}
\end{gathered}
$$

where, $C_{1}, C_{2}$ are constants. Summing up (2.5), (2.9) and (2.10), one obtains (2.6).

\section{§3. The Weak Coupling Limit of the Heisenberg Evolution: Fock Case}

In this section, we shall compute the limit

$$
\lim _{\lambda \rightarrow 0}\left\langle u \otimes \Phi\left(\lambda \int_{S_{1} / \lambda^{2}}^{T_{1} / \lambda^{2}} S_{u} f_{1} d u\right), X^{(\lambda)}\left(t / \lambda^{2}\right) v \otimes \Phi\left(\lambda \int_{S_{2} / \lambda^{2}}^{T_{2} / \lambda^{2}} S_{u} f_{2} d u\right)\right\rangle
$$

where, $X^{(\lambda)}(t)$ is given by $(2.1)$ and $f_{1}, f_{2}, g \in \mathscr{Y}^{\prime}, u, v \in H_{0}, X, D \in B\left(H_{0}\right), T_{1}, S_{1}, T_{2}$, $S_{2} \in \mathbb{R}$ and $t \geq 0$. First of all, notice that, expanding $X^{(\lambda)}\left(t / \lambda^{2}\right)$ in (3.1) according to (2.4), (2.5) and using Lemma (2.1), by the uniform estimate, one can exchange the limit and the summation in this expansion. Therefore,

$$
\begin{aligned}
\lim _{\wedge \rightarrow 0}\langle u \otimes & \left.\otimes\left(\lambda \int_{S_{1} / \lambda^{2}}^{T_{1} / \lambda^{2}} S_{u} f_{1} d u\right), X^{(\lambda)}\left(t / \lambda^{2}\right) v \otimes \Phi\left(\lambda \int_{S_{2} / \lambda^{2}}^{T_{2} / \lambda^{2}} S_{u} f_{2} d u\right)\right\rangle \\
& =\left\langle u \otimes \Phi\left(\chi_{\left|S_{1}, T_{1}\right|} \otimes f_{1}\right),(X \otimes 1) \Phi\left(\chi_{\left|S_{2}, T_{2}\right|} \otimes f_{2}\right)\right\rangle
\end{aligned}
$$




$$
+\sum_{n=1}^{\infty} \sum_{d=0}^{n} \sum_{\sigma \in \lambda_{n}^{0}} \sum_{k=0}^{n} \sum_{1 \leq \mu_{1}<<l_{k} \leq n} \theta(\sigma) \lim _{\lambda \rightarrow 0} \Delta_{n}^{(\lambda)}\left(d, \sigma, j_{1}, \cdots, j_{k}\right)
$$

where, for each $d, \sigma$ and $j_{1}, \cdots, j_{k}$,

$$
\begin{gathered}
\Delta_{n}^{(\lambda)}\left(d, \sigma, j_{1}, \cdots, j_{k}\right):= \\
:=\lambda^{n} \int_{0}^{t / \lambda^{2}} d t_{1} \int_{0}^{t_{1}} d t_{2} \cdots \int_{0}^{t_{n-1}} d t_{n}\left\langle\Phi\left(\lambda \int_{S_{1} / \lambda^{2}}^{T_{1} / \lambda^{2}} S_{u} f_{1} d u\right),\right. \\
\left.A\left(S_{t_{\sigma(1)}} g\right) \cdots A^{+}\left(S_{t_{\sigma(n)}} g\right) \cdots A^{+}\left(S_{t_{\sigma / k}} g\right) \cdots A\left(S_{t_{\sigma(n)}} g\right) \Phi\left(\lambda \int_{S_{2} / \lambda^{2}}^{T_{2} / \lambda^{2}} S_{u} f_{2} d u\right)\right\rangle \\
\left\langle u, D_{n}\left(d, X, j_{1}, \cdots, j_{h}\right) v\right\rangle
\end{gathered}
$$

and for each $\varepsilon \in\{0,1\}^{n}$ satisfying

$$
\varepsilon(j)=\left\{\begin{array}{l}
1, \text { if } j \in\left\{j_{h}\right\}_{h=1}^{h} ; \\
0, \text { if } j \in\{1, \cdots, n\} \backslash\left\{j_{h}\right\}_{h=1}^{h}
\end{array}\right.
$$

and $d=0,1, \cdots, n, D_{n}\left(d, X, j_{1}, \cdots, j_{h}\right)$ is defined as

$$
D_{\varepsilon(1)} \cdots D_{\varepsilon(d)} X D_{\varepsilon(d+1)} \cdots D_{\varepsilon(n)}
$$

In analogy with the decomposition of (5.8a) and (5.8b) in [4], we split each of the terms $\Delta_{n}^{(\lambda)}\left(d, \sigma, j_{1}, \cdots, j_{h}\right)$ into the sum of two terms. Then we determine explicitly the limit of one of these two terms while the other one is shown to vanish as $\lambda \rightarrow 0$. More precisely:

$$
\Delta_{n}^{(\lambda)}\left(d, \sigma, j_{1}, \cdots, j_{h}\right):=I_{n}^{(\lambda)}\left(d, \sigma, j_{1}, \cdots, j_{h}\right)+I_{n}^{(\lambda)}\left(d, \sigma, j_{1}, \cdots, j_{h}\right)
$$

and

$$
\begin{aligned}
& I_{n}^{(\lambda)}\left(d, \sigma, j_{1}, \cdots, j_{k}\right):=\left\langle u, D_{n}\left(d, X, j_{1}, \cdots, j_{k}\right) v\right\rangle \lambda^{n} \int_{0}^{t^{1 / \lambda^{2}}} d t_{1} \int_{0}^{t_{1}} d t_{2} \cdots \int_{0}^{t_{n-1}} d t_{n} \\
& \left\langle\Phi\left(\lambda \int_{S_{1} / \lambda^{2}}^{T_{1} / \lambda^{2}} S_{u} f_{1} d u\right), I_{g}^{\varepsilon}(\sigma) \Phi\left(\lambda \int_{S_{2} / \lambda^{2}}^{T_{2} / \lambda^{2}} S_{u} f_{2} d u\right)\right\rangle \\
& =\sum_{m=0)}^{h \wedge(n-h)} \sum_{\substack{\left.\left.1 \leq q_{1}<<q_{m} \leq n . \\
\mid q_{1}\right\}_{h=1}^{m} \leq l_{l_{h}}\right\}_{h=1}^{h}}} \sum_{\left(\left\{q_{l}, p_{h},\right\}_{h=1}^{m}, \sigma .1\right)}^{\prime} \\
& \left\langle u, D_{n}\left(d, X, j_{1}, \cdots, j_{h}\right) v\right\rangle \lambda^{n} \int_{0}^{1 / \lambda^{2}} d t_{1} \int_{0}^{t_{1}} d t_{2} \cdots \int_{0}^{t_{n-1}} d t_{n}
\end{aligned}
$$

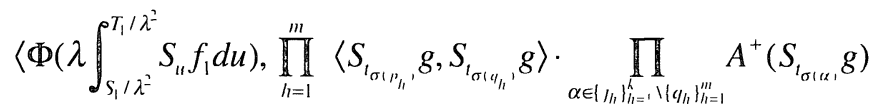

$$
\begin{aligned}
& \left.\prod_{\alpha \in\{1 ., n\} \backslash\left(\mu_{h} h_{h=1}^{h} \cup\left\{p_{h} l_{h=1}^{m}\right)\right.} A\left(S_{t_{\sigma \mid \sigma}} g\right) \Phi\left(\lambda \int_{S_{2} / \lambda^{2}}^{T_{2} / \lambda^{2}} S_{u} f_{2} d u\right)\right\rangle
\end{aligned}
$$

Notice that, for each $\sigma \in \mathcal{Y}_{n}{ }^{0}, 1 \leq q_{1}<\cdots<q_{m} \leq n$ and $1 \leq p_{1}, \cdots, p_{m} \leq n$, there exists a unique $x$ and $1 \leq r_{1}<\cdots<r_{1} \leq m$ such that 


$$
\sigma\left(q_{h}\right)-\sigma\left(p_{h}\right)=1 \text { for } h=r_{1}, \cdots, r_{\text {, }}
$$

and

$$
\sigma\left(p_{h}\right)-\sigma\left(q_{h}\right)=1 \text { for } h \in\{1, \cdots, m\} \backslash\left\{r_{h}\right\}_{h=1}^{r}
$$

Hence, one can write (3.6) as

$$
\begin{aligned}
& I_{n}^{(\lambda)}\left(d, \sigma, j_{1}, \cdots, j_{k}\right)=\sum_{m=0}^{h \wedge(n-h)} \sum_{\substack{\left.1 \leq q_{1}<\cdot<q_{m} \leq n . \\
\left\{q_{h}\right\}_{h=1}^{m} \leq\{\}_{h}\right\}_{h=1}^{h}}} \sum_{\left(\left\{q_{h}, p_{h}\right\}_{h=1}^{m}, \sigma, 1\right)}^{\prime} \\
& \left\langle u, D_{n}\left(d, X, j_{1}, \cdots, j_{k}\right) v\right\rangle \lambda^{n} \int_{0}^{t / \lambda^{2}} d t_{1} \int_{0}^{t_{1}} d t_{2} \cdots \int_{0}^{t_{n-1}} d t_{n} \\
& \left\langle\Phi\left(\lambda \int_{S_{1} / \lambda^{2}}^{T_{1} / \lambda^{2}} S_{u} f_{1} d u\right), \prod_{\left.\left.\alpha \in\right|_{h}\right\}_{h=1}^{\prime}}\left\langle g, S_{t_{\sigma\left(q_{\alpha},\right)^{\prime}} t_{\sigma\left(P_{\alpha}\right)}} g\right\rangle\right.
\end{aligned}
$$

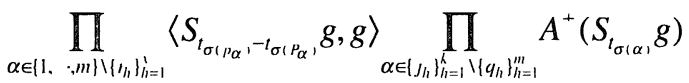

$$
\begin{aligned}
& \left.\prod_{\alpha \in\{1, \ldots\} \backslash\left\{J_{h}\right\}_{h=1}^{h} \cup\left\{p_{h}\right\}_{h=1}^{m}} A\left(S_{t_{\sigma \alpha \alpha},} g\right) \Phi\left(\lambda \int_{S_{2} / \lambda^{2}}^{T_{2} / \lambda^{2}} S_{u} f_{2} d u\right)\right\rangle
\end{aligned}
$$

On the other hand,

$$
\begin{aligned}
& I I_{n}^{(\lambda)}\left(d, \sigma, j_{1}, \cdots, j_{h}\right)
\end{aligned}
$$

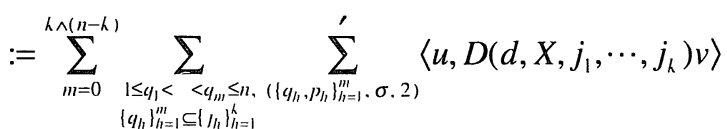

$$
\begin{aligned}
& \lambda^{n} \int_{0}^{t / \lambda^{2}} d t_{1} \int_{0}^{t_{1}} d t_{2} \cdots \int_{0}^{t_{n-1}} d t_{n}\left\langle\Phi\left(\lambda \int_{S_{1} / \lambda^{2}}^{T_{1} / \lambda^{2}} S_{u} f_{1} d u\right),\right.
\end{aligned}
$$

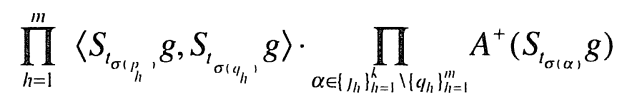

$$
\begin{aligned}
& \left.\prod_{\alpha \in\{1 . . . n\}\left\langle\left\{\mu_{h}\right\}_{h=1}^{h} \cup\left\{p_{h}\right\}_{h=1}^{m}\right)} A\left(S_{t_{\sigma \mid \sigma},} g\right) \Phi\left(\lambda \int_{S_{2} / \lambda^{2}}^{T_{2} / \lambda^{2}} S_{u} f_{2} d u\right)\right\rangle
\end{aligned}
$$

In analogy with Lemma (4.2) in [4], one has the following:

Lemma (3.1). For each $k, n, d \in \mathbf{N}, 1 \leq j_{1}<\cdots<j_{k} \leq n, \sigma \in \mathcal{Y}_{n}^{0}, f_{1}, f_{2}, g \in \mathscr{K}$, $u, v \in H_{0}, D, X \in B\left(H_{0}\right), T_{1}, T_{2}, S_{1}, S_{2} \in \mathbb{R}, t \geq 0$.

$$
\lim _{\wedge \rightarrow 0} I I_{n}^{(\lambda)}\left(d, \sigma, j_{1}, \cdots, j_{k}\right)=0
$$

Proof. With the same argument as in Lemma (1.3), we find:

$$
\left|I I_{n}^{(\lambda)}\left(d, \sigma, j_{1}, \cdots, j_{k}\right)\right| \leq
$$




$$
\begin{aligned}
& \leq \sum_{m=0}^{h \wedge(n-h)} \sum_{\substack{\left.1 \leq q_{1}<<<q_{m} \leq n, . \\
\left\{q_{h}\right\}_{h=1}^{m} \subseteq\{\}_{h}\right\}_{h=1}}} \sum_{\left(\left\{q_{h}, p_{h}\right\}_{h=1}^{m}, \sigma, 2\right)}^{\prime}
\end{aligned}
$$

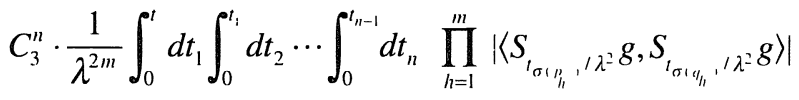

where, $C_{3}$ is a constant. Now it is clear that the integral (3.10) has exactly the same form as the right hand side of (4.22) in [4]. In fact, since in (3.10) only the modulus of the scalar product appears, it does not matter, for this estimate weather we have

$$
\sigma\left(p_{h}\right)<\sigma\left(q_{h}\right) \text { or } \sigma\left(p_{h}\right)>\sigma\left(q_{h}\right)
$$

Therefore, using Lemma (4.2) of [4], we immediately obtain the result.

As the analogue of Theorem (5.1) of [4], we have the following:

Lemma (3.2). For each $n \in \mathbf{N}, f_{1}, f_{2}, g \in \mathscr{K}, u, v \in H_{0}, D, X \in B\left(H_{0}\right), T_{1}, S_{1}$, $T_{2}, S_{2} \in \mathbf{R}$, and $t \geq 0$, one has

$$
\begin{aligned}
& \lim _{\lambda \rightarrow 0}\left\langle u \otimes \Phi\left(\lambda \int_{S_{1} / \lambda^{2}}^{T_{1} / \lambda^{2}} S_{u} f_{1} d u\right), \lambda^{n} \int_{0}^{t / \lambda^{2}} d t_{1} \int_{0}^{t_{1}} d t_{2} \cdots \int_{0}^{t_{n-1}} d t_{n}\right. \\
& \left.\left[V\left(t_{1}\right),\left[V\left(t_{2}\right), \cdots,\left[V\left(t_{n}\right), X \otimes 1\right] \cdots\right]\right] v \otimes \Phi\left(\lambda \int_{S_{2} / \lambda^{2}}^{T_{2} / \lambda^{2}} S_{u} f_{2} d u\right)\right\rangle
\end{aligned}
$$

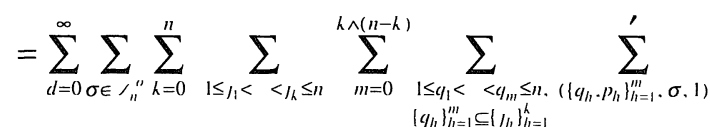

$$
\begin{aligned}
& \left\langle u, D_{n}\left(d, X, j_{1}, \cdots, j_{h}\right) v\right\rangle\left\langle\Psi\left(\chi_{\left[S_{1}, T_{1}\right]} \otimes f_{1}\right), \Psi\left(\chi_{\left[S_{2}, T_{2} \mid\right.} \otimes f_{2}\right)\right\rangle \\
& (g \mid g)_{-}^{\prime} \cdot(g \mid g)_{+}^{m-1} \cdot\left(f_{1} \mid g\right)^{k-m}\left(g \mid f_{2}\right)^{n-h-m} \\
& \int_{\Omega\left(t, \sigma,\left\{q_{h},\right\}_{h=1}^{i},\left\{p_{\alpha}, \alpha \in\{1, . m\} \backslash\left\{\mu_{h}\right\}_{h=1}^{i}\right\}\right)} \\
& \prod_{\left.\left.\alpha \in\{1, . n\} \backslash\left\{\sigma\left(q_{h}\right)\right\}_{h=1}^{\prime} \cup\left\{\sigma\left(p_{\alpha}\right), \alpha \in\{1 ., \ldots\}\right\}\left\{\{\}_{h}\right\}_{h=1}\right\}\right)} d t_{\alpha} \\
& \prod_{\alpha \in\left\{h_{1} \mid\right\}_{h=1}^{\prime} \backslash\left\{q_{h}\right\}_{h=1}^{\prime \prime \prime}} \chi_{\left|S_{1}, T_{1}\right|}\left(t_{\sigma(\alpha)}\right) \cdot \prod_{\alpha \in\{1 ., n\} \backslash\left\{h_{h}\right\}_{h=1}^{\prime} \cup\left\{p_{h}\right\}_{h=1}^{m},} \chi_{\left\{S_{2}, T_{2}\right\}}\left(t_{\sigma(\alpha)}\right)
\end{aligned}
$$

where $\Omega\left(t, \sigma,\left\{q_{h_{h}}\right\}_{h=1}^{\prime},\left\{p_{\alpha}, \alpha \in\{1, \cdots, m\} \backslash\left\{r_{h}\right\}_{h=1}^{\prime}\right\}\right)$ is the $(n-m)$ dimensional subset of $\left\{\left(t_{1}, \cdots, t_{n}\right) ; 0 \leq t_{n} \leq \cdots \leq t_{1} \leq t\right\}$ in which the variables $\left\{t_{\sigma\left(q_{t_{1}}\right.}\right\}_{h=1}^{\prime}$, and $\left\{t_{\sigma\left(q_{\alpha}\right)}\right\}_{\left.\alpha \in\{1, . m\} \backslash h_{h}\right\}_{h=1}^{i},}$ are absent.

Proof. Expanding $\left[V\left(t_{1}\right),\left[V\left(t_{2}\right), \cdots,\left[V\left(t_{n}\right), X \otimes 1\right] \cdots\right]\right]$, by Lemma (3.1), one obtains

$$
\lim _{\lambda \rightarrow 0}\left\langle u \otimes \Phi\left(\lambda \int_{S_{1} / \lambda^{2}}^{T_{1} / \lambda^{2}} S_{u} f_{1} d u\right), \lambda^{n} \int_{0}^{t / \lambda^{2}} d t_{1} \int_{0}^{t_{1}} d t_{2} \cdots \int_{0}^{t_{n-1}} d t_{n}\right.
$$




$$
\begin{aligned}
& \left.\left[V\left(t_{1}\right),\left[V\left(t_{2}\right), \cdots,\left[V\left(t_{n}\right), X \otimes 1\right] \cdots\right]\right] v \otimes \Phi\left(\lambda \int_{S_{2} / \lambda^{2}}^{T_{2} / \lambda^{2}} S_{u} f_{2} d u\right)\right\rangle \\
& =\sum_{d=0}^{n} \sum_{\sigma \in J_{n}^{0}} \sum_{k=0}^{n} \sum_{1 \leq J_{1}<<J_{k} \leq n} \lim _{\lambda \rightarrow 0} I_{n}^{(\lambda)}\left(d, \sigma, j_{1}, \cdots, j_{k}\right)
\end{aligned}
$$

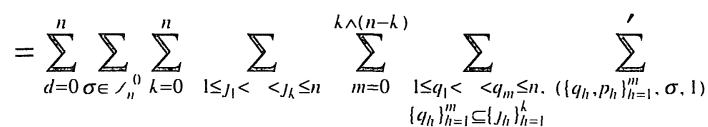

$$
\begin{aligned}
& \left\langle u, D_{n}\left(d, X, j_{1}, \cdots, j_{h}\right) v\right\rangle \lim _{\lambda \rightarrow 0} \lambda^{n} \int_{0}^{t / \lambda^{2}} d t_{1} \int_{0}^{t_{1}} d t_{2} \cdots \int_{0}^{t_{n-1}} d t_{n} \\
& \left\langle\Phi\left(\lambda \int_{S_{1} / \lambda^{2}}^{T_{1} / \lambda^{2}} S_{u} f_{1} d u\right), \prod_{\left.\left.\alpha \in\right|_{h}\right\}_{h=1}^{\prime}}\left\langle g, S_{t_{\sigma\left(q_{\alpha},\right.}, t_{\sigma\left(P_{\alpha}\right)}} g\right\rangle\right.
\end{aligned}
$$

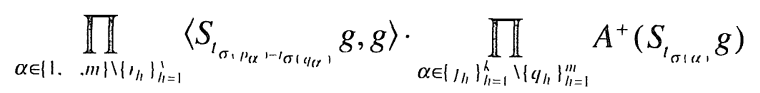

$$
\begin{aligned}
& \left.\underset{\alpha \in\{1, n\} \backslash\left(\zeta_{h h} l_{h=1}^{h} \cup\left\{p_{h}\right\}_{h=1}^{\prime \prime \prime}\right)}{ } A\left(S_{t_{\sigma \alpha \alpha},} g\right) \Phi\left(\lambda \int_{S_{2} / \lambda^{2}}^{T_{2} / \lambda^{2}} S_{u} f_{2} d u\right)\right\rangle
\end{aligned}
$$

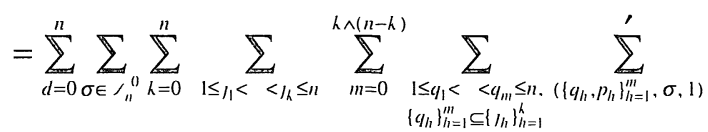

$$
\begin{aligned}
& \left\langle u, D_{n}\left(d, X, j_{1}, \cdots, j_{k}\right) v\right\rangle \lim _{\lambda \rightarrow 0} \lambda^{n} \int_{0}^{t / \lambda^{2}} d t_{1} \int_{0}^{t_{1}} d t_{2} \cdots \int_{0}^{t_{n-1}} d t_{n}
\end{aligned}
$$

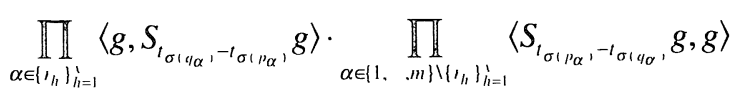

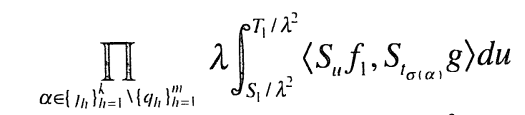

$$
\begin{aligned}
& \prod_{\alpha \in\left\{1 ., n \mid \backslash\left\{h_{h}\right\}_{h=1}^{\infty} \cup\left\{p_{h}\right\}_{h=1}^{m}\right)}\left\langle S_{t_{\sigma(\alpha)}} g, \lambda \int_{S_{2} / \lambda^{2}}^{T_{2} / \lambda^{2}} S_{u} f_{2}\right\rangle d u \\
& \left\langle\Phi\left(\lambda \int_{S_{1} / \lambda^{2}}^{T_{1} / \lambda^{2}} S_{u} f_{1} d u\right), \Phi\left(\lambda \int_{S_{2} / \lambda^{2}}^{T_{2} / \lambda^{2}} S_{u} f_{2} d u\right)\right\rangle
\end{aligned}
$$

Comparing (3.12) with (5.10) in [4], we see that the two expressions are exactly of the same type. So using here the same change of variables, used to establish the equivalence between (5.10) and (5.16) in [4], we see that the role played by the permutations is only in the appearance of terms of the form

$$
\begin{aligned}
& \int_{\left.-s_{\sigma\left(p_{\alpha}\right) / \lambda^{2}}\right)}^{0} d s_{\sigma\left(q_{\alpha}\right)}\left\langle g, S_{\sigma_{\sigma\left(q_{\sigma}\right.},} g\right\rangle \\
& \int_{-s_{\sigma\left(p_{\alpha}\right) / \lambda^{2}}}^{0} d s_{\sigma\left(p_{\alpha}\right)}\left\langle S_{\varsigma_{\sigma\left(p_{\alpha}\right)}} g, g\right\rangle
\end{aligned}
$$

corresponding respectively to $\alpha \in\left\{r_{1}, \cdots, r_{1}\right\}$ and $\alpha \in\{1, \cdots, m\} \backslash\left\{r_{1}, \cdots, r_{1}\right\}$. Thus, introducing the notations: 


$$
\int_{0}^{\infty}\langle g, S, g\rangle d s=:(g \mid g)_{+}, \int_{-\infty}^{0}\langle g, S, g\rangle d s=:(g \mid g)_{-}
$$

the Lemma follows immediately.

Combining together Lemma (2.1), Lemma (3.1) and Lemma (3.2), we have

Lemma (3.3) For each $\left.f_{1}, f_{2}, g \in\right\rangle^{\prime}, u, v \in H_{0}, D, X \in B\left(H_{0}\right), T_{1}, T_{2}, S_{1}, S_{2}$ $\in \mathbf{R}, t \geq 0$, the limit

$$
\lim _{\lambda \rightarrow 1)}\left\langle u \otimes \Phi\left(\lambda \int_{S_{1} / \lambda^{2}}^{T_{1} / \lambda^{2}} S_{u} f_{1} d u\right), X^{(\lambda)}\left(t / \lambda^{2}\right) v \otimes \Phi\left(\lambda \int_{S_{2} / \lambda^{2}}^{T_{2} / \lambda^{2}} S_{u} f_{2} d u\right)\right\rangle
$$

exists and is equal to

$$
\begin{aligned}
& \sum_{n=0}^{\infty} \sum_{d=0}^{n} \sum_{\sigma \in>_{n}^{\prime \prime}} \sum_{k=0}^{n} \sum_{1 \leq \mu_{1}<<l_{k} \leq n} \theta(\sigma) \sum_{m=0}^{h \wedge(n-h)} \sum_{1 \leq q_{1}<<q_{m !}<n,} \sum_{\left(\left\{q_{h}, p_{h}\right\}_{h=1}^{\prime \prime}, \sigma, 1\right)}^{\prime} \\
& \left\langle u, D_{n}\left(d, X, j_{1}, \cdots, j_{h}\right) v\right\rangle\left\langle\Psi\left(\chi_{\left.\mid S_{1}, T_{1}\right]} \otimes f_{1}\right), \Psi\left(\chi_{\left[S_{2}, T_{2}\right]} \otimes f_{2}\right)\right\rangle \\
& (g \mid g)_{-}^{\prime} \cdot(g \mid g)_{+}^{m-1} \cdot\left(f_{1} \mid g\right)^{h-m}\left(g \mid f_{2}\right)^{n-h-m} \\
& \int_{\Omega\left(1, \sigma,\left\{q_{\eta_{1}},\right\}_{h=1},\left\{\left\{p_{\alpha}, \alpha \in\left[1,, m \mid \backslash\left\langle y_{h}\right\}_{h=1}^{\prime}\right\}\right)\right.\right.} \\
& \prod_{\left.\alpha \in\{1 . . n\} \backslash\left\{\sigma\left(u_{1, h}\right)\right\}_{h=1} \cup\left\{\sigma\left(p_{\alpha}\right), \alpha \in\{1 . . m\} \backslash\left(\{\}_{h}\right\}_{h=1}\right\}\right)} d t_{\alpha}
\end{aligned}
$$

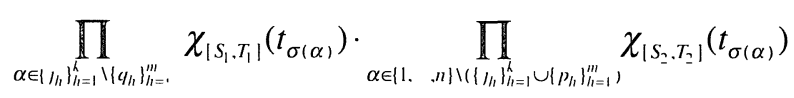

\section{\$4. The Quantum Langevin Equation}

In the section, we shall study the relation between the limit (3.16) and the solution of the quantum stochastic differential equation (q.s.d.e.)

$$
U(t)=1+\int_{0}^{t}\left(D \otimes d A^{+}(s, g)-\left(D^{+} \otimes d A(s, g)-(g \mid g)_{-} D^{+} D \otimes 1 d s\right) U(s)\right.
$$

on $H_{0} \otimes \Gamma\left(L^{2}(\mathbb{R}) \otimes \#\right)$. Put

$$
X(t)=U(t)(X \otimes 1) U^{+}(t), t \geq 0
$$

then, by Ito's formula,

$$
\begin{gathered}
d X(t)=(d U(t)) \cdot(X \otimes 1) \cdot U^{+}(t)+U(t) \cdot(X \otimes 1) \cdot d U^{+}(t) \\
+(d U(t)) \cdot(X \otimes 1) \cdot d U^{+}(t) \\
=\left(D \otimes d A^{+}(t, g)-D^{+} \otimes d A(t, g)-(g \mid g)_{-} D^{+} D \otimes 1 d t\right) X(t)+ \\
X(t)\left(D^{+} \otimes d A(t, g)-D \otimes d A^{+}(t, g)-(g \mid g)_{+} D^{+} D \otimes 1 d t\right) \\
+\left(D \otimes d A^{+}(t, g)-D^{+} \otimes d A(t, g)-(g \mid g)_{-} D^{+} D \otimes 1 d t\right) X(t) \\
\left(D^{+} \otimes d A(t, g)-D \otimes d A^{+}(t, g)-(g \mid g)_{+} D^{+} D \otimes 1 d t\right)
\end{gathered}
$$




$$
\begin{gathered}
+\left(D \otimes d A^{+}(t, g)-D^{+} \otimes d A(t, g)-(g \mid g)_{-} D^{+} D \otimes 1 d t\right) X(t) \\
\left(D^{+} \otimes d A(t, g)-D \otimes d A^{+}(t, g)-(g \mid g)_{+} D^{+} D \otimes 1 d t\right)
\end{gathered}
$$

In other words, the process $X(t)$ satisfies the quantum Langevin equation:

$$
\begin{gathered}
d X(t)=\left(D \otimes d A^{+}(t, g)-D^{+} \otimes d A(t, g)-(g \mid g)_{-} D^{+} D \otimes 1 d t\right) X(t)+ \\
X(t)\left(D^{+} \otimes d A(t, g)-D \otimes d A^{+}(t, g)-(g \mid g)_{+} D^{+} D \otimes 1 d t\right) \\
+(g \mid g) \cdot\left(D^{+} \otimes 1\right) X(t)(D \otimes 1) d t \\
X(0)=X \otimes 1
\end{gathered}
$$

For each $X, D \in B\left(H_{0}\right), f_{1}, f_{2}, g \in K, T_{1}, S_{1}, T_{2}, S_{2} \in \mathbf{R}, t \geq 0$, it is clear that there exists an operator $\hat{X}(t)$ on $H_{0}$ such that for each $u, v \in H_{0}$,

$$
\langle u \otimes \hat{X}(t) v\rangle=\left\langle u \otimes \Psi\left(\chi_{\left|s_{1} \cdot T_{1}\right|} \otimes f_{1}\right), X(t) v \otimes \Psi\left(\chi_{\left|s_{2}, T_{2}\right|} \otimes f_{2}\right)\right\rangle
$$

Denote

$$
F(u, v, t)=\langle u \otimes \hat{X}(t) v\rangle
$$

then,

$$
F(u, v, 0)=\langle u, X v\rangle\left\langle\Psi\left(\chi_{\left[S_{1}, T_{1}\right]} \otimes f_{1}\right), \Psi\left(\chi_{\left[S_{2}, T_{2} \mid\right.} \otimes f_{2}\right)\right\rangle
$$

Moreover,

$$
\begin{gathered}
F(u, v, t)=F(u, v, 0)+\int_{0}^{t}\left\langle u \otimes \Psi\left(\chi_{\left|S_{1}, T_{1}\right|} \otimes f_{1}\right),\left[(D \otimes 1)\left(1 \otimes d A^{+}(s, g)\right)+\right.\right. \\
\left.\left.\left(-D^{+} \otimes 1\right)(1 \otimes d A(s, g))+(g \mid g)_{-}\left(-D^{+} D \otimes 1\right) d s\right] X(s) v \otimes \Psi\left(\chi_{\left[S_{2}, T_{2}\right]} \otimes f_{2}\right)\right\rangle \\
+\int_{0}^{t}\left\langle u \otimes \Psi\left(\chi_{\left|S_{1}, T_{1}\right|} \otimes f_{1}\right), X(s)\left[(-D \otimes 1)\left(1 \otimes d A^{+}(s, g)\right)+\right.\right. \\
\left.\left.\left(D^{+} \otimes 1\right)(1 \otimes d A(s, g))+(g \mid g)_{+}\left(-D^{+} D \otimes 1\right) d s\right] v \otimes \Psi\left(\chi_{\left[S_{2}, T_{2}\right]} \otimes f_{2}\right)\right\rangle \\
+\int_{0}^{t}\left\langle u \otimes \Psi\left(\chi_{\left|S_{1}, T_{1}\right|} \otimes f_{1}\right),(g \mid g)\left(D^{+} \otimes 1\right) X(s)(D \otimes 1) v \otimes \Psi\left(\chi_{\left[S_{2}, T_{2}\right]} \otimes f_{2}\right)\right\rangle \\
=F(u, v, 0)+\int_{0}^{t} d s\left(f_{1} \mid g\right) \chi_{\left|S_{1}, T_{1}\right|}(s) F\left(D^{+} u, v, s\right)+\left(f_{1} \mid g\right) \chi_{\left.\mid S_{1}, T_{1}\right]}(s) F(u,-D v, s) \\
+\left(g \mid f_{2}\right) \chi_{\left.\mid S_{2}, T_{2}\right]}(s) F(-D u, v, s)+\left(g \mid f_{2}\right) \chi_{\left.\mid S_{2}, T_{2}\right]}(s) F\left(u, D^{+} v, s\right) \\
+(g \mid g)_{-} F\left(-D^{+} D u, v, s\right)+(g \mid g)_{+} F\left(u,-D^{+} v, s\right)+(g \mid g) F(D u, D v, s)
\end{gathered}
$$

It is clear that the integral equation (4.8) has a unique solution global in $t$ since $D$ is bounded. So, in order to prove that the limit (3.16) is equal to $F(u, v, t)$ for each $t \geq 0$, it is sufficient to prove that the limit (3.16) satisfies (4.8). To this goal, denote the limit (3.16) $G(u, v, t)$ and notice that, because of the uniform convergence of the series in (3.16), this is an a.e. differentiable function on $\mathbb{R}^{+}$. Moreover, for each fixed $\lambda$

$$
\frac{d}{d t}\left\langle u \otimes \Phi\left(\lambda \int_{S_{1} / \lambda^{2}}^{T_{1} / \lambda^{2}} S_{u} f_{1} d u\right), X^{(\lambda)}\left(t / \lambda^{2}\right) \nu \otimes \Phi\left(\lambda \int_{S_{2} / \lambda^{2}}^{T_{2} / \lambda^{2}} S_{u} f_{2} d u\right\rangle\right.
$$


$=\left\langle u \otimes \Phi\left(\lambda \int_{S_{1} / \lambda^{2}}^{T_{1} / \lambda^{2}} S_{u} f_{1} d u\right),(-i) \frac{1}{\lambda} \cdot\left[V_{g}\left(t / \lambda^{2}\right), X^{(\lambda)}\left(t / \lambda^{2}\right)\right] v \otimes \Phi\left(\lambda \int_{S_{2} / \lambda^{2}}^{T_{2} / \lambda^{2}} S_{u} f_{2} d u\right)\right\rangle$

and

$$
\begin{gathered}
(-i) \frac{1}{\lambda} \cdot\left[V_{g}\left(t / \lambda^{2}\right), X^{(\lambda)}\left(t / \lambda^{2}\right)\right] \\
=\frac{1}{\lambda} \cdot\left(\left(D \otimes A^{+}\left(S_{t / \lambda^{2}} g\right)-D^{+} \otimes A\left(S_{t / \lambda^{2}} g\right)\right) \cdot X^{(\lambda)}\left(t / \lambda^{2}\right)\right. \\
\left.-X^{(\lambda)}\left(t / \lambda^{2}\right) \cdot\left(D \otimes A^{+}\left(S_{t / \lambda^{2}} g\right)-D^{+} \otimes A\left(S_{t / \lambda^{2}} g\right)\right)\right) \\
=\frac{1}{\lambda} \cdot\left(D \otimes A^{+}\left(S_{t / \lambda^{2}} g\right) \cdot X^{(\lambda)}\left(t / \lambda^{2}\right)-\left(D^{+} \otimes 1\right) \cdot X^{(\lambda)}\left(t / \lambda^{2}\right) \cdot 1 \otimes A\left(S_{t / \lambda^{2}} g\right)\right. \\
-\left(D^{+} \otimes 1\right) \cdot\left[1 \otimes A\left(S_{t / \lambda^{2}} g\right), X^{(\lambda)}\left(t / \lambda^{2}\right)\right] \\
-1 \otimes A^{+}\left(S_{t / \lambda^{2}} g\right) \cdot X^{(\lambda)}\left(t / \lambda^{2}\right) \cdot(D \otimes 1)+X^{(\lambda)}\left(t / \lambda^{2}\right) \cdot D^{+} \otimes A\left(S_{t / \lambda^{2}} g\right) \\
\left.-\left[X^{(\lambda)}\left(t / \lambda^{2}\right), 1 \otimes A^{+}\left(S_{t / \lambda^{2}} g\right)\right] \cdot(D \otimes 1)\right)
\end{gathered}
$$

In analogy with the proof of Lemma (6.2) in [4], one gets easily:

$$
\begin{gathered}
\lim _{\lambda \rightarrow 0}\left\langle u \otimes \Phi\left(\lambda \int_{S_{1} / \lambda^{2}}^{T_{1} / \lambda^{2}} S_{u} f_{1} d u\right),\right. \\
\frac{1}{\lambda}\left(D \otimes A^{+}\left(S_{t / \lambda^{2}} g\right) \cdot X^{(\lambda)}\left(t / \lambda^{2}\right)-\left(D^{+} \otimes 1\right) \cdot X^{(\lambda)}\left(t / \lambda^{2}\right) \cdot 1 \otimes A\left(S_{t / \lambda^{2}} g\right)\right. \\
\left.\left.-1 \otimes A^{+}\left(S_{t / \lambda^{2}} g\right) \cdot X^{(\lambda)}\left(t / \lambda^{2}\right) \cdot(D \otimes 1)-X^{(\lambda)}\left(t / \lambda^{2}\right) \cdot D^{+} \otimes A\left(S_{t / \lambda^{2}} g\right)\right)\right) \\
v \otimes \Phi\left(\lambda \int_{S_{2} / \lambda^{2}}^{T_{2} / \lambda^{2}} S_{l t} f_{2} d u\right\rangle \\
=\left(f_{1} \mid g\right) \chi_{\left[S_{1}, T_{1}\right]}(t) G\left(D^{+} u, v, t\right)+\left(f_{1} \mid g\right) \chi_{\left[S_{1}, T_{1}\right]}(t) G(u,-D v, t) \\
+\left(g \mid f_{2}\right) \chi_{\left[S_{2}, T_{2}\right]}(t) G(-D u, v, t)+\left(g \mid f_{2}\right) \chi_{\left[S_{2}, T_{2}\right]}(t) G\left(u, D^{+} v, t\right)
\end{gathered}
$$

Furthermore, one has

$$
\begin{gathered}
\left(D^{+} \otimes 1\right) \cdot\left[1 \otimes A\left(S_{t / \lambda^{2}} g\right), X^{(\lambda)}\left(t / \lambda^{2}\right)\right] \\
=\sum_{n=1}^{\infty}\left(D^{+} \otimes 1\right)(-i)^{n} \lambda^{n} \int_{0}^{t / \lambda^{2}} d t_{1} \int_{0}^{t_{1}} d t_{2} \cdots \int_{0}^{t_{n-1}} d t_{n} \\
{\left[1 \otimes A^{+}\left(S_{t / \lambda^{2}} g\right),\left[V_{g}\left(t_{1}\right),\left[V_{g}\left(t_{2}\right), \cdots,\left[V_{g}\left(t_{n}\right), X \otimes 1\right] \cdots\right]\right]\right]} \\
=\sum_{n=1}^{\infty}\left(D^{+} \otimes 1\right)(-i)^{n} \lambda^{n} \int_{0}^{t / \lambda^{2}} d t_{1} \int_{0}^{t_{1}} d t_{2} \cdots \int_{0}^{t_{n-1}} d t_{n} \\
\left(\left[1 \otimes A\left(S_{t / \lambda^{2}} g\right), V_{g}\left(t_{1}\right)\right] \cdot\left[V_{g}\left(t_{2}\right), \cdots,\left[V_{g}\left(t_{n}\right), X \otimes 1\right] \cdots\right]\right. \\
-\left[V_{g}\left(t_{2}\right), \cdots,\left[V_{g}\left(t_{n}\right), X \otimes 1\right] \cdots\right] \cdot\left[1 \otimes A\left(S_{t / \lambda^{2}} g\right), V_{g}\left(t_{1}\right)\right] \\
\left.+V_{g}\left(t_{1}\right) \cdot\left[1 \otimes A\left(S_{t / \lambda^{2}} g\right),\left[V_{g}\left(t_{2}\right), \cdots,\left[V_{g}\left(t_{n}\right), X \otimes 1\right] \cdots\right]\right]\right) \\
=\left(D^{+} \otimes 1\right)(-i) \frac{1}{\lambda} \int_{0}^{t} d t_{1} \cdot\left(\left[1 \otimes A\left(S_{t / \lambda^{2}} g\right), V_{g}\left(t_{1} / \lambda^{2}\right)\right] \cdot X^{(\lambda)}\left(t_{1} / \lambda^{2}\right)\right. \\
-X^{(\lambda)}\left(t_{1} / \lambda^{2}\right) \cdot\left[1 \otimes A\left(S_{t / \lambda^{2}} g\right), V_{g}\left(t_{1} / \lambda^{2}\right)\right] \\
-\left[1 \otimes A\left(S_{t / \lambda^{2}} g\right), X^{(\lambda)}\left(t_{1} / \lambda^{2}\right)\right] \cdot V_{g}\left(t_{1}\right)
\end{gathered}
$$




$$
\begin{gathered}
\left.+V_{g}\left(t_{1}\right) \cdot\left[1 \otimes A\left(S_{t / \lambda^{2}} g\right), X^{(\lambda)}\left(t_{1} / \lambda^{2}\right)\right]\right) \\
=\left(D^{+} \otimes 1\right)(-i) \frac{1}{\lambda} \int_{0}^{t} d t_{1} \cdot\left(i(D \otimes 1)\left\langle S_{t / \lambda^{2}} g, S_{t_{1} / \lambda^{2}} g\right\rangle \cdot X^{(\lambda)}\left(t_{1} / \lambda^{2}\right)\right. \\
-X^{(\lambda)}\left(t_{1} / \lambda^{2}\right) \cdot i(D \otimes 1)\left\langle S_{t / \lambda^{2}} g, S_{t_{1} / \lambda^{2}} g\right\rangle \\
-\left[1 \otimes A\left(S_{t / \lambda^{2}} g\right), X^{(\lambda)}\left(t_{1} / \lambda^{2}\right)\right] \cdot V_{g}\left(t_{1}\right) \\
\left.+V_{g}\left(t_{1}\right) \cdot\left[1 \otimes A\left(S_{t / \lambda^{2}} g\right), X^{(\lambda)}\left(t_{1} / \lambda^{2}\right)\right]\right)
\end{gathered}
$$

By the proof of Lemma (6.4) in [4], one gets

$$
\begin{gathered}
\lim _{\lambda \rightarrow 0}\left\langle u \otimes \Phi\left(\lambda \int_{S_{1} / \lambda^{2}}^{T_{1} / \lambda^{2}} S_{u} f_{1} d u\right),\left(D^{+} \otimes 1\right)(-i) \frac{1}{\lambda} \int_{0}^{t} d t_{1} i(D \otimes 1)\right. \\
\left\langle S_{t / \lambda^{2}} g, S_{t_{1} / \lambda^{2}} g\right\rangle \cdot X^{(\lambda)}\left(t_{1} / \lambda^{2}\right) v \otimes \Phi\left(\lambda \int_{S_{2} / \lambda^{2}}^{T_{2} / \lambda^{2}} S_{u} f_{2} d u\right\rangle \\
=(g \mid g)_{-} \cdot G\left(D^{+} D u, v, t\right) \\
\lim _{\lambda \rightarrow 0}\left\langle u \otimes \Phi\left(\lambda \int_{S_{1} / \lambda^{2}}^{T_{1} / \lambda^{2}} S_{u} f_{1} d u\right),\left(D^{+} \otimes 1\right)(-i) \frac{1}{\lambda} \int_{0}^{t} d t_{1}\right. \\
\left.X^{(\lambda)}\left(t_{1} / \lambda^{2}\right) \cdot i(D \otimes 1)\left\langle S_{t / \lambda^{2}} g, S_{t_{1} / \lambda^{2}} g\right\rangle v \otimes \Phi\left(\lambda \int_{S_{2} / \lambda^{2}}^{T_{2} / \lambda^{2}} S_{u} f_{2} d u\right)\right\rangle \\
=(g \mid g)_{-} \cdot G(D u, D v, t)
\end{gathered}
$$

and

$$
\begin{gathered}
\lim _{\lambda \rightarrow 0}\left\langle u \otimes \Phi\left(\lambda \int_{S_{1} / \lambda^{2}}^{T_{1} / \lambda^{2}} S_{u} f_{1} d u\right),\left(D^{+} \otimes 1\right)(-i) \frac{1}{\lambda} \int_{0}^{t} d t_{1}\right. \\
\left(\left[1 \otimes A\left(S_{t / \lambda^{2}} g\right), X^{(\lambda)}\left(t_{1} / \lambda^{2}\right)\right] \cdot V_{g}\left(t_{1}\right)\right. \\
+V_{g}\left(t_{1}\right) \cdot\left[1 \otimes A\left(S_{t / \lambda^{2}} g\right), X^{(\lambda)}\left(t_{1} / \lambda^{2}\right)\right] v \otimes \Phi\left(\lambda \int_{S_{2} / \lambda^{2}}^{T_{2} / \lambda^{2}} S_{u} f_{2} d u\right\rangle \\
=0
\end{gathered}
$$

Similarly, we obtain

$$
\begin{gathered}
\lim _{\lambda \rightarrow 0}\left\langle u \otimes \Phi\left(\lambda \int_{S_{1} / \lambda^{2}}^{T_{1} / \lambda^{2}} S_{u} f_{1} d u\right),\left(D^{+} \otimes 1\right)(-i) \frac{1}{\lambda}\right. \\
\left.\left(-\left[X^{(\lambda)}\left(t / \lambda^{2}\right), 1 \otimes A^{+}\left(S_{t / \lambda^{2}} g\right)\right] \cdot(D \otimes 1)\right) v \otimes \Phi\left(\lambda \int_{S_{2} / \lambda^{2}}^{T_{2} / \lambda^{2}} S_{u} f_{2} d u\right)\right\rangle \\
=-(g \mid g)_{+} G\left(u, D^{+} D v, t\right)+(g \mid g)_{+} G(D u, D v, t)
\end{gathered}
$$

Summing up (4.9), (4.10), (4.11), (4.12), (4.14), (4.15) and (4.16), one has

$$
\begin{aligned}
& \lim _{\lambda \rightarrow 0} \frac{d}{d t}\left\langle u \otimes \Phi\left(\lambda \int_{S_{1} / \lambda^{2}}^{T_{1} / \lambda^{2}} S_{u} f_{1} d u\right), X^{(\lambda)}\left(t / \lambda^{2}\right) v \otimes \Phi\left(\lambda \int_{S_{2} / \lambda^{2}}^{T_{2} / \lambda^{2}} S_{u} f_{2} d u\right)\right\rangle \\
& \quad=\left(f_{1} \mid g\right) \chi_{\left|S_{1} \cdot T_{1}\right|}(t) G\left(D^{+} u, v, t\right)+\left(f_{1} \mid g\right) \chi_{\left|S_{1}, T_{1}\right|}(t) G(u,-D v, t) \\
& \quad+\left(g \mid f_{2}\right) \chi_{\left|S_{2}, T_{2}\right|}(t) G(-D u, v, t)+\left(g \mid f_{2}\right) \chi_{\left|S_{2}, T_{2}\right|}(t) G\left(u, D^{+} v, t\right)
\end{aligned}
$$




$$
+(g \mid g)_{-} G\left(-D^{+} D u, v, t\right)+(g \mid g)_{+} G\left(u,-D^{+} D v, t\right)+(g \mid g) G(D u, D v, t)
$$

Since each term in the series expansion of

$$
\frac{d}{d t}\left\langle u \otimes \Phi\left(\lambda \int_{S_{1} / \lambda^{2}}^{T_{1} / \lambda^{2}} S_{u} f_{1} d u\right), X^{(\lambda)}\left(t / \lambda^{2}\right) v \otimes \Phi\left(\lambda \int_{S_{2} / \lambda^{2}}^{T_{2} / \lambda^{2}} S_{u} f_{2} d u\right)\right\rangle
$$

has a form similar to the terms obtained from the expansion of

$$
\left\langle u \otimes \Phi\left(\lambda \int_{S_{1} / \lambda^{2}}^{T_{1} / \lambda^{2}} S_{u} f_{1} d u\right), X^{(\lambda)}\left(t / \lambda^{2}\right) v \otimes \Phi\left(\lambda \int_{S_{2} / \lambda^{2}}^{T_{2} / \lambda^{2}} S_{u} f_{2} d u\right)\right\rangle
$$

we can use the uniform estimate here obtaining

$$
\begin{gathered}
G(u, v, t)=G(u, v, 0)+\lim _{\lambda \rightarrow 0} \int_{0}^{t} \frac{d}{d s}\left\langle u \otimes \Phi\left(\lambda \int_{S_{1} / \lambda^{2}}^{T_{1} / \lambda^{2}} S_{u} f_{1} d u\right),\right. \\
X^{(\lambda)}\left(s / \lambda^{2}\right) \nu \otimes \Phi\left(\lambda \int_{S_{2} / \lambda^{2}}^{T_{2} / \lambda^{2}} S_{u} f_{2} d u>d s\right. \\
=G(u, v, 0)+\int_{0}^{t}\left(\left(f_{1} \mid g\right) \chi_{\left[S_{1}, T_{1}\right]}(s) G\left(D^{+} u, v, s\right)+\left(f_{1} \mid g\right) \chi_{\left[S_{1}, T_{1}\right]}(s) G(u,-D v, s)\right. \\
+\left(g \mid f_{2}\right) \chi_{\left[S_{2}, T_{2}\right]}(s) G(-D u, v, s)+\left(g \mid f_{2}\right) \chi_{\left[S_{2}, T_{2}\right]}(s) G\left(u, D^{+} v, s\right) \\
\left.+(g \mid g)_{-} G\left(-D^{+} D u, v, s\right)+(g \mid g)_{+} F\left(u,-D^{+} D v, s\right)+(g \mid g) G(D u, D v, s)\right) d s
\end{gathered}
$$

On the other hand, it is clear that

$$
G(u, v, 0)=F(u, v, 0)
$$

thus, comparing (4.18) with (4.8) we obtain the following theorem

Theorem (4.1). For each $f_{1}, f_{2}, g \in \aleph^{\prime}, u, v \in H_{0}, D, X \in B\left(H_{0}\right), T_{1}, T_{2}, S_{1}, S_{2}$ $\in \mathbb{R}, t \geq 0$,

$$
\begin{aligned}
& \lim _{\lambda \rightarrow 0}\left\langle u \otimes \Phi\left(\lambda \int_{S_{1} / \lambda^{2}}^{T_{1} / \lambda^{2}} S_{u} f_{1} d u\right), X^{(\lambda)}\left(t / \lambda^{2}\right) v \otimes \Phi\left(\lambda \int_{S_{2} / \lambda^{2}}^{T_{2} / \lambda^{2}} S_{u} f_{2} d u\right\rangle\right. \\
& =\left\langle u \otimes \Psi\left(\chi_{\left|S_{1} \cdot T_{1}\right|} \otimes f_{1}\right), U(t)(X \otimes 1) U^{+}(t) v \otimes \Psi\left(\chi_{\left|S_{2}, T_{2}\right|} \otimes f_{2}\right)\right\rangle
\end{aligned}
$$

where, $U(t)$ is the unique solution of q.s.d.e. (4.1).

Finally, combining $\S 1, \S 2, \S 3$ and $\S 4$, we have the following:

Theorem (4.2)。For each $f_{1}, f_{2}, g \in . \check{Y}^{\prime}, u, v \in H_{0}, D, X \in B\left(H_{0}\right), T_{1}, T_{2}, S_{1}, S_{2}$ $\in \mathbb{R}, t \geq 0$ and $Q \geq 1$, the limit

$$
\lim _{\lambda \rightarrow 0}\left\langle u \otimes W_{Q}\left(\lambda \int_{S_{1} / \lambda^{2}}^{T_{1} / \lambda^{2}} S_{u} f_{1} d u\right) \Phi_{Q}, X^{(\lambda)}\left(t / \lambda^{2}\right) v \otimes W_{Q}\left(\lambda \int_{S_{2} / \lambda^{2}}^{T_{2} / \lambda^{2}} S_{u} f_{2} d u\right) \Phi_{Q}\right\rangle
$$

exists and is equal to

$$
\left\langle u \otimes W_{1 \otimes Q}\left(\chi_{\mid S_{1}, T_{1} !} \otimes f_{1}\right) \Psi_{1 \otimes Q}, U(t)(X \otimes 1) U^{+}(t) v \otimes W_{1 \otimes Q}\left(\chi_{\left|s_{2}, T_{2}\right|} \otimes f_{2}\right) \Psi_{1 \otimes Q}\right\rangle
$$


where,

$$
\left\langle\Psi_{1 \otimes Q}, W_{1 \otimes Q}(\xi \otimes f) \Psi_{1 \otimes Q}\right\rangle=\exp \left(-\frac{1}{2}\langle\xi \otimes f,(1 \otimes Q)(\xi \otimes f)\rangle\right) .
$$

for $\xi \in L^{2}(\mathbf{R})$ and $U(t)$ is the unique solution of the quantum stochastic differential equation (1.45).

\section{References}

[1] Accardi, L., On the quantum Feynman-Kac formula, Rend. Sem. Math. Fis. Uni. Polit. Milano, 48 (1078), 135-179

[2] - Noise and dissipation in quantum theory. Rev. Math. Phys., 2, No 2 (1990), 127-176.

[3] Frigerio, A. and Lu, Y.G., on the weak coupling limit problem. Quantum probability and its applications IV, Springer, Lecture Notes in Math., 1396 (1988), 20-58.

[4] The weak coupling limit as a quantum functional central limit, Commu. Math. Phys., 131 (1991), 537-570.

[5] Davies, E. B., Markovian master equation, Comm. Math. Phys., 39 (1974), 91-110.

[6] Quantum Theory of Open Systems, Academic Press, London and New York, 1976.

[7] De Smedt, P., Dür, D., Lebowitz, J.L. and Liverani, C., Quantum system in contact with a thermal environment: rigorous treatment of a simple model, Comm. Math. Phys. 120(1988), 195-231.

[8] Hudson, R. L. and Parthasarathy, K. R., Quantum Ito's formula and stochastic evolutions, Comm . Math. Phys. 91 (1985), 301-323.

[9] Pulé, J. V., The Bloch Equation, Comm. Math. Phys., 38 (1974), 241-256.

[10] van Hove, L., Quantum mechanical pertubations giving rise to a statistical transpost equation, Physica, 21 (1995), 517-540.

[11] Accardi, L. and Parthasarathy K. R., Stochastic Calculus on local algebras. Quantum Probability and Applications II, Springer-Verlag, Lecture Notes in Math., 1136 (1995), 9-23.

[12] Applebaum, D., Quasi-free stochastic evolutions. Accardi, L., and von Walden-fels, W.(Eds.): Quantum Probability and Applications II, Springer, Lecture Notes in Math., 1136(1985), 46-56. 